\title{
The (Un)detectability of Absolute Newtonian Masses
}

\author{
Niels C.M. Martens*
}

18 April 2019

Forthcoming in Synthese

\begin{abstract}
Absolutism about mass claims that mass ratios obtain in virtue of absolute masses. Comparativism denies this. Dasgupta (2013) argues for comparativism about mass, in the context of Newtonian Gravity. Such an argument requires proving that comparativism is empirically adequate. Dasgupta equates this to showing that absolute masses are undetectable, and attempts to do so. This paper develops an argument by Baker to the contrary: absolute masses are in fact empirically meaningful, that is detectable (in some weak sense). Additionally, it is argued that the requirement of empirical adequacy should not be cashed out in terms of undetectability in the first place. The paper closes by sketching the possible strategies that remain for the comparativist. Along the way a framework is developed that is useful for thinking about these issues: Ozma games - how could one explain to an alien civilisation what an absolute mass is?
\end{abstract}

Keywords - physical quantities - comparativism - absolutism - mass - Newtonian gravity detectability

\section{Defining Absolutism \& Comparativism}

What does it mean for a physical determinable to be dimensionful? In order to start answering this question, this paper evaluates the case study of mass, as it features within Newtonian Gravity (NG). For simplicity, we will assume standard NG to comprise an ontology of $n$ particles in a flat (pace Knox [1]) Newtonian space and time, with an ideology ${ }^{1}$ of absolute distances, velocities and finite, positive, non-zero masses, governed by Newton's three laws and the Law of Universal Gravitation ${ }^{2}$, and a principle of equivalence between inertial and gravitational mass ${ }^{3}$. Dasgupta notices the following interesting aspect of the property of having mass:

\footnotetext{
*RWTH Aachen University, Institute for Theoretical Particle Physics and Cosmology, Sommerfeldstrasse 16, 52074 Aachen, Germany, martens@physik.rwth-aachen.de, +49 24180 27054, Orcid:0000-0002-2839-1387

${ }^{1}$ Here I am using 'ontology' and 'ideology' in the Quinean sense [2]. Roughly, ontology refers to the (primitive) objects, and ideology to their (primitive) properties.

${ }^{2}$ What laws the textbook equations exactly represent will be discussed in $\S 6.2$.

${ }^{3}$ In the context of $\mathrm{NG}$, inertial and gravitational mass are usually merely empirically identified, but still theoretically or conceptually distinguished. It would then be interesting to consider whether one could be an absolutist - defined below - about inertial mass but a comparativist - defined below - about gravitational mass, and vice versa. In this paper we will however follow the current literature in bracketing out such a possibility, by considering only a single mass determinable.
} 
"The property of having mass is a determinable that appears to have two kinds of determinates. On the one hand, we naturally think that something with mass has a determinate intrinsic property, a property it has independently of its relationships with other material bodies. But we also think that things with mass stand in various determinate mass relationships with one another, such as $x$ being more massive than $y$ or $x$ being twice as massive as $y . "[3$, p.105]

It is the central aim of this paper to determine how these two determinates - mass relations and absolute masses - relate, metaphysically speaking.

The standard formulation of NG seems to explicitly model the absolute ${ }^{4}$ mass of each particle, by a numerical value associated with that particle (where that value depends on the unit chosen, say kilograms), rather than modeling only mass ratios between particles. This particle is $1 \mathrm{~kg}$, that particle is $4 \mathrm{~kg}$, etc. Naive literalism about our physical theories seems to suggest a metaphysical commitment to that absolute mass scale, and even a metaphysical priority of those absolute masses over the mass ratios.

(Weak, Metaphysical) Absolutism: The determinate absolute masses ground the mass relations.

I take the notion of 'to ground' to be synonymous with 'to be true or to obtain in virtue of', 'to be metaphysically prior to' and 'to explain'. In other words, according to absolutism, the mass relations are explained by the more fundamental absolute masses. 'Explain' should here not be understood in the causal sense, but in the metaphysical sense: $\mathrm{P}$ holds in virtue of the metaphysical facts that make it count as P. Thus, a causal explanation of 'Niels played underwater hockey last Sunday evening' would be that I had received an invite, that I saw on YouTube how amazing this sport is ${ }^{5}$, that I biked to the swimming pool, etc. A metaphysical explanation, on the other hand, should mention that what makes it count that Niels was playing underwater hockey last Sunday evening. In this case that might be the combination of facts that I was underwater, wearing a mask, in a team of six people, shooting pucks in the opponent's goal, etc.

Perhaps it is fair to say that absolutism is the received view. Lewisian Humean Supervenience, in particular, has taken this view on board by positing a spatiotemporal mosaic with intrinsic masses (and charges) sprinkled onto it $^{6}$. Roberts claims that absolutism surely is the commonsense position [4]. Views that count as absolutism have been defended by Armstrong [16,17], Mundy [18] and Lewis [19].

That being said, there is also a strong opposing tradition. Mach's operational definition of mass has been highly influential (among physicists) [20]. He defined mass relations, specifically mass ratios, (via acceleration ratios) and considered his work done; no need for a further operational definition of absolute masses, let alone granting them metaphysical priority over the mass ratios. We may call this comparativism about mass.

\footnotetext{
${ }^{4}$ Although Dasgupta takes it to be an essential feature of absolute masses that they are intrinsic, I side with Roberts [4] (and Sider [5]) in taking the crucial distinction between absolute masses and mass relations to be that the former are monadic properties, and the latter dyadic properties (i.e. binary relations). After all, regularity comparativism [6] grounds absolute masses in a complete four-dimensional mosaic of spatiotemporal relations and fundamental mass ratios, making them as extrinsic as they could possibly be, whilst leaving their monadicity untouched. The Higgs mechanism for generating inertial masses in the Standard Model of Particle Physics seems to similarly generate monadic masses that are nevertheless extrinsic, as the mass emerges from an interaction with, or immersion in the Higgs field [7].

${ }^{5}$ It is! You should check it out online.

${ }^{6}$ But see $[6,8-15]$.
} 
(Weak, Metaphysical) Comparativism: The determinate mass relations are not grounded in determinate absolute masses.

(We will follow Mach in this paper by assuming that the relevant mass relationships ${ }^{7}$ are the scale-independent ones (see Baker for a justification [21]), which under suitable constraints [22, Ch.1\&5] can be interpreted as mass ratios ${ }^{8}$.) Similarly, Field's nominalist research programme, as presented in Science without Numbers $[23]^{9}$, was and is highly influential (among philosophers), and has comparativism as a by-product: its fundamental nominalist predicates are a betweenness and a congruence relation. Russell discussed it in his The Principles of Mathematics [25]. Ellis defended it in 1966 [26]. It was central to Bigelow, Pargetter \& Armstrong's 1988 paper [27] (see also Bigelow \& Pargetter's book [28]). Arntzenius briefly touches upon the topic in his Space, Time, and Stuff [29] in 2012, but it is not until 2013 that the terms 'absolutism' and 'comparativism' are coined by Dasgupta [3] - in a paper that will be the focal point of the current paper. Eddon's paper [30] in that same year cites Dasgupta's paper but does not yet adopt the terminology. Also of relevance is Perry's PhD thesis on the metaphysics of physical quantities [31]. The most recent defense of comparativism is a manuscript by Roberts [4].

Why would someone place (fundamental) importance only on mass ratios? For one thing, the numerical values used to model masses in the standard formulation of NG are conventional. That is because this modeling comprises not only a numerical value but also an arbitrary unit. A unit is nothing but a relation in disguise. Often we choose the unit ' $\mathrm{kg}$ ', which uses the International Prototype of the Kilogram in Paris as the standard reference object ${ }^{10}$, but any other reference object would do, in principle. A different choice of unit means that the masses of each object are represented by a different numerical value. This arbitrariness should perhaps not be surprising. Is there anything intrinsically ' 5 -ish' about (the mass of) a square object of $5 \mathrm{~kg}$ in the same sense as there is something intrinsically '4ish' about its shape (i.e. the number of its corners)? No. After all, $5 \mathrm{~kg}$ is equivalent to 11 pounds, whereas the property of having 4 corners is not equivalent to having a non-four number of 'anythings'.

Note that comparativism is silent on the status of the absolute masses. It is often held in conjunction with either the view that facts about (the quantities used to represent) absolute masses hold in virtue of the mass relationships together with a convention, such as deciding to refer to the mass of International Prototype of the Kilogram in Paris as $1 \mathrm{~kg}$, or a view that simply denies the existence or meaningfulness of absolute masses. It is important to realise that these are claims that go beyond comparativism. For instance, elsewhere I discuss a view that denies both of these two claims - the absolute masses are grounded in the mass relations plus other non-conventional non-mass facts, namely a complete four-dimensional mosaic of spatiotemporal relations - but is nevertheless a version of comparativism $[6]^{11}$.

\footnotetext{
${ }^{7}$ Strictly speaking, in first order logic, there is just one mass relation, and several instantiations of that single relation. In this paper, as in most of the literature, when we talk about mass relationships, this is short for several instantiations of this single mass relation. Why this makes sense will become more clear in $\S 3$, where I will advocate defining absolute mass(es) and the mass 'relation(s)' using group theory.

${ }^{8}$ By this I do not mean to rule out nominalistic versions of comparativism - such as Field's approach which uses primitive congruence predicates as mass relations [23] - in favour of quantitative approaches - such as the numerical mass ratios used by, for instance, Dasgupta. I merely intend to exclude relations such as, in quantitative terms, ' $\mathrm{x}$ is $2 \mathrm{~kg}$ more massive than $\mathrm{y}$ '.

${ }^{9}$ See also his 1985 paper [24].

${ }^{10}$ On the 20th of May 2019 the Bureau International des Poids et Mesures will redefine the $\mathrm{kg}$ in terms of natural constants.

${ }^{11}$ Although I end up arguing against this view, that is not because it is not a coherent comparativist view.
} 
If defined as above, weak comparativism is the denial of weak absolutism, which makes this pair of views mutually exhaustive ${ }^{12}$. However, some of the literature tends to focus on stronger versions, either implicitly or explicitly ${ }^{13}$. They do not only concern the relative metaphysical priority/fundamentality of the two types of determinates, but also claim that the metaphysically prior determinate is itself ungrounded.

\section{Strong (Metaphysical) Absolutism:}

1. Weak (Metaphysical) Absolutism

2. Primitivism ${ }^{14}$ : Mass is fundamental (within the theory, i.e. Newtonian Gravity). That is, the determinate absolute masses are not themselves grounded in anything further (described within that same theory).

\section{Strong (Metaphysical) Comparativism:}

\section{Weak (Metaphysical) Comparativism}

2. Primitivism: Mass is fundamental (within the theory). That is, the determinate mass relationships are not themselves grounded in anything further (described within that same theory).

These two stronger definitions are not mutually exhaustive: both are inconsistent with a denial of the second part, namely with the view that mass (both absolute masses and mass relationships) is not fundamental, not even within Newtonian Gravity. This third position within the resulting trilemma may be called reductionism about mass ${ }^{15}$. Our discussion will touch upon this position in $§ 8.3$ - for more elaborate discussions against two types of reductionism see refs. [6,34]. When mentioning absolutism/comparativism in this paper without any further specification, the distinction between the weak and the strong metaphysical versions will either be irrelevant, or it will be clear from the context that the weak metaphysical version is intended.

In $\S 2$ I develop a framework that is useful for thinking about absolutism-comparativism debates - Ozma Games - which indicates that we need a more precise definition of mass ratios

\footnotetext{
12 That is if we assume the existence of mass ratios. It is an interesting question whether we truly need mass ratios (within NG), or whether they are merely an artefact of our representation in terms of quantities. As far as I am aware all of the comparativism literature assumes the existence of mass ratios, so we will do so as well (for now).

${ }^{13}$ Dasgupta defines absolutism as "the view that the most fundamental facts about material bodies vis-à-vis their mass include facts about which intrinsic mass they posses" [3, p.105], and comparativism as "the view that the most fundamental facts about material bodies vis-à-vis their mass just concern how they are related in mass, and all other facts about their mass hold in virtue of those relationships" [3, p.105-6]. As they stand, these statements could be interpreted either in the weak sense or in, what I call below, the strong sense, depending on whether 'most fundamental' is taken to be a statement about absolute fundamentality, or whether 'most fundamental vis-à-vis their mass' is read as being about relative fundamentality. Since Dasgupta also claims that the absolutist believes that "intrinsic masses are fundamental" [3, p.105], he is best interpreted as explicitly discussing the strong versions of absolutism and comparativism. However, nothing in the rest of his paper turns out to hinge on considering the strong rather than the weak versions. In particular, none of the arguments gives any reason to go beyond the weak versions. Hence, as explained in the text, I believe it best in general to focus on the weak versions, especially since only they are mutually exhaustive.

${ }^{14}$ Dees calls this Quantity Primitivism, but in the current paper 'quantity' is a highly technical notion ( $\$ 3$ ), distinct from my equally technical notion of 'magnitude', which is presumably what Dees had in mind [32]. I am choosing for Primitivism rather than Magnitude Primitivism though, because in the case of strong comparativism the primitivism refers not to the magnitudes but the ratios.

${ }^{15} \mathrm{An}$ analogous distinction in the space debate is made in Martens [33].
} 
and especially of absolute masses $(\S 3)$ in order to avoid a common confusion regarding what exactly is at stake in this debate $(\S 4)$. This finally allows me to introduce the main argument in favour of comparativism in $\S 5$, its main premise being the claim that comparativism is empirically adequate. By analogy with the substantivalism-relationalism debate, we may wish to cash out this requirement in terms of 1) uniform mass scaling being a symmetry, 2) absolute masses being undetectable, or 3) comparativism being able to reproduce the correct set of empirically possible worlds. After outlining Dasgupta's comparativist proposal in $\S 6$, I present my interpretation of Dasgupta's reasons for believing that, on this proposal, absolute masses are undetectable, and his comparativism therefore empirically adequate ( $\$ 7)$. The first result of this paper is to develop an argument by Baker to show that absolute masses are empirically meaningful, and thus detectable, albeit in a very weak sense $\S 8$. The second result ( $\S 9)$ is then to show, mainly because this sense of detectability is so weak, that the requirement of empirical adequacy should not be cashed out in terms of the undetectability of absolute masses, but instead as comparativism's ability to reproduce the correct set of empirically possible worlds (which is developed elsewhere [22, Ch.3] [35]). I then translate these results back to the Ozma Games framework in $\S 10$. Although these results provide a severe challenge for comparativism, they do not by themselves conclusively rule in favour of absolutism. I sketch the remaining strategies open to comparativism in $\S 11$.

\section{Operationalising Mass: Ozma Games}

A helpful framework for thinking about the debate between absolutism and comparativism about mass is obtained by adapting Gardner's Ozma problem for handedness [36]. Gardner asks whether we could convey the meaning of 'left(-handedness)' to aliens, solely by sending them a signal in morse code, without being allowed to ostensively refer to handed structures on the night sky. Invoking, for instance, the fact that human hearts are on the left side of the body will not be of any help, since this is contingent: for all we know the alien hearts are in fact on the right side - the laws of nature allow it. The same applies to the right-handed twist of our DNA helices, etc. This problem can easily be generalised to properties such as shape and mass. Equivalently, we could send the aliens a 4-kg left-handed glove (with 5 fingers) and enquire after its properties.

If we ask the aliens for the shape of the object, that is the number of fingers, no problems arise. No comparativist position on (this aspect of) shape could even be formulated, and once a numerical system - say the decimal system - is agreed upon there is also no ambiguity arising from the way the number of fingers is represented. The answer is simply ' 5 '.

A problem does seem to arise if we enquire after the absolute mass. We would not expect the aliens to give the 'correct' answer, ' $4 \mathrm{~kg}$ ', when we inquire after the mass of the object. We would expect something like '10,000 zorb', where, so they explain after we communicate our non-understanding of this answer, 1 zorb is the mass of their reference mass in their capital city. It seems that there is no way of doing better at communicating the mass property than by expressing it comparatively. That just seems to be what it is for a physical determinable (such as mass) to be dimensionful. After all, there is nothing intrinsically '4-ish' about the glove or its mass, whereas there is something intrinsically ' 5 -ish' about it. This difference is explained by the number of fingers of a glove being a 1) discrete and 2) dimensionless quantity; this allows us to simply count the corners. Mass, at least in (the standard formulation of) NG, is a continuous and dimensionful notion, hence the absolute mass of an object cannot simply be 
counted in the same way. Would a property that is only discrete but not dimensionless suffice to provide said contrast with Newtonian mass? That depends. Take for instance electric charge, which we believe to be quantised in quanta of $\frac{1}{3} e$. The existence of this smallest quantum removes the arbitrariness that a conventional unit such as $\mathrm{kg}$ has - although we are of course still free to choose any unit we want, however 'unnatural' - but does not imply that we can count the electric charge of an individual object in the same sense in which we can count its corners. This would be the case if all electrically charged particles 'featured' a discrete number of quanta of $\frac{1}{3} e$, in the same way as a glove features a discrete number of fingers. Current theories tell us that this is not the case: the up quark, for instance, has a charge of $\frac{2}{3} e$, but this is not taken to mean that it carries 2 packages of electrical charge, just that it carries 1 package with a numerical (dimensionful) value that is twice as large as the minimum possible value $^{16}$. Similarly, a property that resembles mass only by being continuous but not by being dimensionful would also fail to provide said contrast. An example would be (solid) angle. It is true that representing an angle as a fraction of the maximally possible angle (in that space) would make it somewhat objective (i.e. less conventional than the unit of mass), but an angle of $5 \%$ of the maximal angle is still not ' 5 -ish' in the way that a glove is. It is thus indeed both the discreteness and the dimensionlessness of the number of fingers of a glove that makes it intrinsically ' 5 -ish' in a way that (particles with) properties that lack either of these featuressuch as absolute mass which lacks both - are not.

This story illustrates in a more vivid way the point hinted at in the previous section, that there is nothing intrinsically '4-ish' about the (mass of the) glove. The numerical values associated with the absolute masses are not unique, but unit-dependent, and therefore conventional. And if absolute masses are to be real let alone (relatively) fundamental, it seems to be a necessary condition for their values to be well-defined (i.e. unique).

Even if one could overcome this worry by somehow arguing for a privileged unit of mass, there is an explanatory worry about the numerical values of the mass in that fundamental unit. Field [23] points out that physical theories should provide an explanation without appeal to extraneous, causally irrelevant entities ${ }^{17,18}$. (Platonic) numbers are such causally inert entities.

If, as at first blush appears to be the case, we need to invoke some real numbers like $6.67 \times 10^{-11}$ (the gravitational constant in $\mathrm{m}^{3} / \mathrm{kg}^{-1} / \mathrm{s}^{-2}$ ) in our explanation of why the moon follows the path that it does, it isn't because we think that that real number plays a role as a cause of the moon's moving that way; it plays a very different role in the explanation than electrons play in the explanation of the working of electric devices. The role it plays is as an entity extrinsic to the process to be explained, an entity related to the process to be explained only by a function (a rather arbitrarily chosen function at that). Surely then it would be illuminating if we could show that a purely intrinsic explanation of the process was possible,

\footnotetext{
${ }^{16}$ Perhaps distance (or time) as it features in theories of discrete spacetime such as causal set theory [37] would be a more interesting example. In this case, spatial (or temporal) extension boils down to counting the spacetime atoms. However, in such a case it is questionable whether distance (or time) is still truly a dimensionful determinable.

${ }^{17}$ Arntzenius and Dorr echo this thought [29, Ch.8].

${ }^{18}$ One might argue that the comparativist faces that same objection: a relation such as ' $\mathrm{x}$ is twice as massive as y' refers indirectly to the platonic number two. This is true for the quantitative approach to comparativism of, for instance, Bigelow \& Pargetter [27] and Dasgupta [3], but not for congruence-based comparativism, as advocated famously by Field himself [23]. In the next section we will see that the same type of solution exists for the absolutist.
} 
an explanation that did not invoke functions to extrinsic and causally irrelevant entities. [23, p.43]

Additionally, there would be an epistemological worry: we would neither have direct access to, nor acquaintance with, those 'absolute mass values' associated with this privileged unit, whereas we do have relatively direct access to shape via touch or almost directly via sight. What would it mean to 'see a mass'? It is not as if a number appears on our visual image whenever we look at a massive object.

It thus seems that the best we could do, when trying to convey the concept of mass to aliens, is to convey the mass ratios, for instance, but not necessarily, by relating it to a proto-mass in their or our capital city. The Ozma Mass Problem seems to suggest that comparativism about mass is true.

\section{Magnitudes vs. Quantities}

This conclusion is premature. These worries derive from failing to distinguish between physical magnitudes and the numerical quantities used to represent them ${ }^{19,20}$ - a failure that will keep recurring in the remainder of this paper, as I believe it to be one of the crucial confusions that misdirects many authors towards comparativism. The absolutist is committed to the magnitudes (grounding the mass relations), not the quantities. Compare this again with shape: that a certain function from the corners of a polygon to $\mathbb{R}^{+}$gives us, say, the number 5 , is a mathematical fact about that object (plus that function) but does not imply that there is a (fundamental) Platonic numerical value (quantity) or even magnitude (as defined below) attached to that object. Absolutism merely claims that there are brute absolute mass magnitudes (which ground the mass relations), where I define physical magnitudes as (meta)physical properties which mirror some of the structure of $\mathbb{R}^{+}$without in fact being a Platonistic version of the numerals in $\mathbb{R}^{+}$or a mapping to those numerals, that is without in fact being quantities. Magnitudes are thus the continuous version of the number of corners of a polygon-shaped object. That they can be represented by quantities in a multitude of ways stems from the mere fact that multiplying $\mathbb{R}^{+}$by an overall scalar is a non-trivial automorphism.

More precisely, I define physical magnitudes as follows. The determinate physical magnitudes falling under one physical determinable comprise a set of monadic properties with cardinality $2^{\aleph_{0}}$. On the elements of this set we place two ${ }^{21}$ structures: 1) a total order, and 2) an associative concatenation structure which is to be interpreted as addition - it fixes where the sum of several magnitudes 'fits into the total order'"22. In the case of mass, the first structure

\footnotetext{
${ }^{19}$ Perhaps this failure to distinguish magnitudes and quantities is sufficient to undermine what Dasgupta calls 'The Objection from Kilograms' [3, §5] (which is, in contrast to the worries discussed in the previous section of this paper, seen as an objection against comparativism).

${ }^{20}$ Hall similarly points out that physical determinates, such as absolute mass, are not linguistic entities but (monadic or polyadic) magnitudes [8].

${ }^{21}$ One may wonder whether we also need multiplicative structure, as the gravitational force depends on the product of two masses (and in order to define the Active Leibniz Mass Scaling defined below). One response would be to argue that this structure is part of the laws and not the masses, although it could be retorted that we could make the same move for all the structure that we do attribute to masses here (cf. Dees [32]). Fortunately, in standard NG we need not worry about products of masses, since we have assumed an equivalence principle between empirical and inertial mass, such that the final equation that governs the dynamics, $a=G m / r^{2}$, does not contain any product of masses.

${ }^{22}$ The abovementioned constraints on mass relations required to prove the representation theorem are exactly
} 
is associated (operationally speaking) with comparing the masses of two objects by putting one massive object on each scale of a balance. Adding the second structure corresponds to adding several massive objects on each scale of the balance. In the case of electric charge these structures generate a totally ordered group, since electric charge can be neutral or negative, thereby providing an identity element and an inverse for every element in the set. In the case of mass this generates a totally ordered semi-group. It does not form a totally ordered group since there is no inverse (no negative masses) and no identity element (no zero mass, at least not in NG).

As it stands, these structures render all absolute masses qualitatively identical ${ }^{23}$. Then, in order for the laws to know in each possible world which determinate magnitude they are "latching onto', the determinate magnitudes need to be endowed with non-qualitative, transworld identities (i.e. these identities are required for the forces to be well-defined, in the sense of uniquely matching up instances of initial conditions, including masses, with, say, accelerations) - more on this in Section 9. In the case of strong absolutism these identities will be primitive; in the case of weak absolutism these transworld identities will presumably be reducible to the transworld identities of the properties to which the absolute masses reduce. (Primitive) non-qualitative, transworld identity of properties is usually referred to as quidditism, but it is often ambiguous whether quidditism is supposed to refer to transworld identities of the determinates or the determinables, so we will specify the current view as determinate quidditism or quidditism about absolute mass determinates ${ }^{24}$.

Perhaps surprisingly, the mass ratios of the comparativist have a similar structure. They also consist in a set of determinate properties, again with cardinality $2^{\aleph_{0}}$. These properties are however binary relations, rather than monadic properties. These deteminate properties are again 1) totally ordered, and 2) obey an associative concatenation structure, but this concatenation structure is to be interpreted as multiplication ${ }^{25}$. It includes the relation ' $\ldots$ is as massive as ...'- or in quantitative terms '... stands in a 1:1 mass ratio to ...'-which forms the identity element, and also includes an inverse for every mass ratio. Hence, the mass ratios form a totally ordered group. As with absolute masses, the comparativist mass ratios need to be endowed with non-qualitative, transworld identities, in order for the laws to know in each possible world which determinate mass relation they are 'latching onto'. ${ }^{26}$

In order to be a realist about absolute masses in Newtonian Gravity we should thus be aware that a mathematical theory representing magnitudes necessarily has to choose one of the

the constraints that ensure that the mass relations can be represented by monadic properties with these two structures.

${ }^{23}$ It is for this reason that kinematic comparativism, as defined in the next section, is true.

${ }^{24}$ Insofar as determinables are grounded in determinates, it would only be a small leap to argue from determinate quidditism to transworld identities of determinables.

${ }^{25}$ These structures correspond nicely to Weyl's operational definition of (inertial) mass [38, 39].

${ }^{26}$ The easiest way to see this is by realising that there is no qualitative distinction between a first set of binary relations that obey these structures, and a second set obtained from the first by squaring each relation-call this a Leibniz Mass Ratio Squaring. (I would like to thank Zee Perry for pointing this out to me.) Note that it is for this same reason that the mass ratio structure, as defined in the text, does not fix a sum over mass ratios-as this would not be invariant under a Leibniz Squaring. Although a structure that defines such a sum is usually implicitly assumed for the rational numbers, i.e. quantities, it is not yet fixed for the much sparser concept of mass ratios defined in the text. Such a sum over mass ratios would require extra structure [35]. Here we see yet another reason why it is dangerous to confuse numerical quantities with the absolute magnitudes or mass ratios that they represent. Moreover, focusing on magnitudes and mass ratios with their sparse structure rather than numerical quantities with their rich structure is in line with the desideratum of metaphysical parsimony that is central to the debate between absolutism and comparativism (cf. §5) [35]. 
conventional homomorphisms of $\mathbb{R}+$, the set of quantities, onto those magnitudes, but it is the magnitude and not the quantity that one should be realist about. ${ }^{27}$ Realising this confusion dissolves the worries raised at the end of the previous section.

In summary, the absolutism-comparativism debate concerns magnitudes and relations (which are related in a many-to-one fashion), and not quantities and relations. Unfortunately magnitudes need to be represented by quantities, which are related to magnitudes in a many-to-one fashion. (Conversely, if a single set of quantities is chosen (in a way that is consistent with a set of mass ratios), this also still leaves open many different sets of absolute magnitudes that those quantities may be mapped to.) I believe, as we will see, that it is this double (or even triple) many-to-one relation that wrongly guides some authors towards comparativism about mass. ${ }^{28}$

\section{Kinematic \& Dynamic Comparativism}

The representational redundancy - of quantities when representing magnitudes - that was exposed in the previous section stems from some epistemological cousin of (metaphysical) comparativism being trivially true.

Consider first the analogous substantivalism-relationalism debate about the metaphysics of space(time). Leibnizian relationalists often point out that distances and velocities can only be described or expressed, non-dynamically, by refering to some material reference body. Being told the position of your car with respect to the 'centre of the universe' or the spatial point labelled 'Tushar' is not going to help you find it. Let us call this kinematic relationalism. ${ }^{29}$

An analogous story applies to our case. Mass is a dimensionful determinable. As such, the following epistemological cousin of comparativism is true of it:

Kinematic Comparativism: ${ }^{30}$ For any dimensionful determinable, such as mass, the magnitude predicated of any particle can only be reported or expressed, non-dynamically, in terms of how this magnitude relates to the magnitude of another particle having the same determinable property.

In fact, we should take it not merely as a feature but as a definition of the dimensionfulness of a determinable that it is kinematically comparative. Only dimensionless determinables, such as the number of fingers of a glove, can be expressed in a more direct way. We might call them kinematically absolute.

It is because at the kinematic level mass is comparative that on the representational level it must be represented by a quantity times a unit. A unit is thus merely a relation - to a standard

\footnotetext{
${ }^{27} \mathrm{cf.}[5, \S 3.2]$.

${ }^{28}$ See, for instance, Roberts $[4, \S 5]$.

${ }^{29}$ I adapt this terminology (as well as 'dynamic relationalism', see main text) from Huggett [40], who uses the term 'kinematic relativity'. However, 1) the notion of relativity is easily confused-in the context of the philosophy of space - with Einstein's notion of the relativity of simultaneity, and 2) kinematic relativity is the epistemological or kinematic version of Leibnizian (metaphysical) relationalism, making 'kinematic relationalism' the more obvious choice.

${ }^{30}$ The distinction bewteen kinematic comparativism and dynamic comparativism (see main text) is not only analogous to Huggett's distinction (cf. fn.29), but also closely related to Van Cleve's 'existentially relational' vs. 'referentially relational' properties [41, p.209] (which echoes Brentano's distinction between the Relativ and the Relativlich [42, p.272] [41, p.209]) and Bennett's 'reference by telling' vs. 'reference by showing' (which is relevant for the Ozma games discussed in the main text) [43] [44, p.132] [45, p.15].
} 
reference body (pace Dasgupta [46]) - in disguise. This in turn necessitates a conventional choice of the specific unit (i.e. an arbitrarily deisgnated reference body that is represented by the quantity 1), which introduces representational redundancy. But does this redundancy at the representational level have any implications for the redundancy of absolute masses at the metaphysical level (or at least for their metaphysical priority over mass ratios)?

A naive argument that directly infers metaphysical comparativism from kinematic comparativism goes as follows: if we can only express mass ratios and not (non-conventional) absolute masses, then that must be because there simply are only mass ratios. ${ }^{31}$ That this argument is invalid is most easily seen by returning to our anology with the spatiotemporal debate. ${ }^{32}$ Substantivalists have to agree with kinematic relationalism: when reporting the location of one's car one needs to relate it to other material objects, as it cannot be meaningfully reported by relating it to the centre of substantival space. But they insist that it does not follow that physics, that is the evolution of these relational notions, depends only on these relational distances (and higher-order time-derivatives) (i.e. dynamic relationalism) - a fortiori it does not follow that metaphysical relationalism is true. An independent argument is needed for dynamic relationalism, and (the mere (prima facie) possibility of) Newton's bucket and globes ${ }^{33}$ reveal(s) exactly this logical and physical gap between kinematic and dynamic relationalism. Even though we can meaningfully express the location (and acceleration) of the water only with respect to the bucket (or other material objects, but not with respect to absolute space), its subsequent behaviour could be construed as nevertheless depending on its rotation with respect to substantival space (pace Barbour [49]).

Define the analogue of dynamic relationalism as follows:

Dynamic Comparativism: Physics depends only on the mass ratios, not on further absolute masses grounding those ratios. In other words, metaphysical comparativism is empirically adequate.

What we mean by 'empirically adequate' will have to be made more precise in the remainder of this paper, but an intuitive understanding suffices to see that the mere fact that masses need to be expressed via comparisons does not mean that physics does not depend on the absolute masses over and above the mass comparisons. Kinematic comparativism does not imply dynamic comparativism (and therefore a fortiori does not imply metaphysical comparativism), just as kinematic relationalism does not imply dynamic relationalism. An independent argument would be needed for dynamic comparativism, and it is the main aim of this paper to show that that argument does not exist in the context of Newtonian Gravity.

\section{The Argument for Comparativism}

In this section I will present the general schema for the comparativist argument. The subsequent two sections will reconstruct Dasgupta's specific comparativist proposal and his specific interpretation of the general schema for the comparativist argument. Dasgupta's approach is then criticised in two further sections.

\footnotetext{
${ }^{31}$ It seems that Mach had something like this in mind [34], and that Roberts is invoking something like this $[4, \mathrm{p} .4,12]$.

${ }^{32}$ Zanstra [47] suggests that Berkeley, Aristotle and Copernicus seem to make the invalid argument of inferring (metaphysical) relationalism from kinematic relationalism. At the very least they are confusing the two notions.

${ }^{33}$ In Newton's Scholium to the Definintions in his Principia [48].
} 
An argument for the empirical adequacy of metaphysical comparativism is what the comparativist needs. But even if this can be found, metaphysical absolutism would still be consistent with the empirical adequacy of comparativism. After all, the absolutist does not deny the reality of mass ratios, only their (relative) fundamentality. It would be true though that in that case the absolute masses would be metaphysically redundant (over and above the mass ratios). A comparativist who is motivated by metaphysical parsimony may then invoke some Occamist norm that urges us to get rid of such redundant properties (i.e. Occam's Razor). The comparativist's problem with their opponent is thus, in general, not that they believe that the absolutist position is incoherent ${ }^{34}$ or inconsistent, but just that their commitment to absolute masses grounding mass ratios is unnecessary and on top of that unnecessarily 'expensive' (metaphysically speaking). In analogy with the Occamist arguments that are usually invoked against absolute velocities in NG, the general schema for the comparativist argument that seems prevalent in the literature is then:

\begin{tabular}{ll}
$\mathbf{P}_{\text {dyn }}$ & $\begin{array}{l}\text { Dynamic Comparativism: (Metaphysical) comparativism is } \\
\text { empirically adequate. }\end{array}$ \\
$\mathbf{P}_{\text {occ-par }}$ & $\begin{array}{l}\text { Occamist norm: All other things being equal (i.e. } \mathrm{P}_{\text {dyn }} \\
\text { assuming the empirical adequacy of absolutism }\end{array}{ }^{36}$ ), we should favour \\
& theories that are metaphysically more parsimonious. \\
& $\begin{array}{l}\text { (Metaphysical) comparativism about mass is metaphysically more } \\
\text { parsimonious than (metaphysical) absolutism. }\end{array}$ \\
\hline $\mathbf{C}$ & (Metaphysical) comparativism about mass is favoured over (meta- \\
physical) absolutism.
\end{tabular}

\subsection{Three Approaches to Empirical Adequacy}

It will once again prove fruitful to model our discussion of the empirical adequacy of comparativism on the template provided by the analogous debate about the metaphysics of space. Three popular approaches to interpreting arguments in favour or against empirical adequacy dominate the literature. It is generally accepted that Static and Kinematic Leibniz Shifts, if doing anything at all, provide a challenge for the substantivalist, whereas Rotational Dynamic Leibniz Shifts (i.e. Newton's bucket experiment and globes thought experiment) threaten the empirical adequacy of relationalism.

On the first approach, the symmetry approach, the former two Leibniz shifts are interpreted as symmetries of Newtonian Gravity, whereas the latter is not. The Occamist norm is then explicated as a direct symmetry-to-(un)reality inference, for instance via Saunders' Invariance Principle: "only quantities ${ }^{37}$ invariant under exact symmetries are real" [50, p.1-2].

On the second approach, the undetectability approach, the first two types of shifts are interpreted as exposing the undetectability of absolute positions and velocities, whereas the

\footnotetext{
${ }^{34}$ Unless one combines comparativism - specifically a version that is anti-realist about absolute masses (see Section 9) - with a verification theory of meaning.

${ }^{35}$ As argued elsewhere $[22, \S 1.2 .3]$, this argument is not valid. For all other things to be equal we presumably also need to check whether comparativism (at least) equals absolutism when it comes to explanatory power and other relevant extra-empirical criteria. In this paper I question the soundness of this argument.

${ }^{36}$ In the domain of applicability of the theory.

${ }^{37}$ 'Quantities' is used more colloquially by Saunders than the technical definition in the current paper.
} 
Rotational Dynamic Leibniz Shift reveals the detectability of absolute rotational accelerations. The Occamist norm should then be interpreted as a razor for undetectable notions.

The third approach, the possibility checking approach [51-53], checks whether the theory generates the correct set of empirically possible worlds (or at the very least includes a possible world that is empirically equivalent to the actual world). From each relationalist world, uncountably many metaphysically distinct substantival worlds can be generated via the three types of Leibniz Shifts. Ideally, there should be at least one metaphysically distinct possible world associated with each empirically distinct possible world allowed by (substantivalist) Newtonian Gravity. If relationalism satisfies this criterion-which seems to be the case for worlds related by the first two types of shift, but not the latter type of shift-but substantivalism generates 'too many' metaphysical possibilities, an Occamist norm can then be invoked against substantivalism.

The thought experiment central to the current debate is not whether it would make a difference if God had placed all the matter in the universe 1 meter to the left, or boosted it with $1 \mathrm{~m} / \mathrm{s}$, etc., but whether it would have made a difference if God had decided to, say, double the absolute masses of all the particles in the universe. In other words, the relevant transformation that threatens the empirical adequacy of comparativism is what we may call a Leibniz Scaling:

(Active) Leibniz (Mass) Scaling: A uniform scalar multiplication ${ }^{38}$ of each of the absolute mass magnitudes of all the particles, ceteris paribus (i.e. whilst keeping everything else unchanged).

(What makes this transformation 'active' will be discussed below.) The threat to comparativism that is the focus of this paper concerns the question of whether a Leibniz Scaling, which changes only the absolute masses but leaves the mass relations and everything else invariant, produces an empirical difference. If it does, and I will argue that it does, this suggests that comparativism is not empirically adequate.

As in the space debate, there are three approaches to interpreting and responding to the Leibniz Scaling argument against (or in favour of) the empirical adequacy of comparativism.

A comparativist using the symmetry approach will claim that Leibniz Scaling is a symmetry of Newtonian Gravity, and therefore, by the Invariance Principle, absolute masses are not (objectively) real (and a fortiori not more fundamental than mass ratios). An absolutist will deny that it is a symmetry.

On the undetectability approach, the empirical adequacy of comparativism is equated to the absolute masses being undetectable over and above the mass relations.

The possibility checking approach explicates a sufficient condition for empirical adequacy as comparativism's ability to generate the correct set of empirically possible worlds. From the previous section we now realise that the representation theorem between absolute

\footnotetext{
${ }^{38}$ Does it make sense to multiply magnitudes (rather than the quantities that represent them) by a scalar (Cian Dorr, personal communication)? We could perhaps respond to this question by adding extra (multiplicative) structure to the absolute masses, but it seems that that structure is not needed within Newtonian Gravity (cf. fn. 21), and it would reduce the metaphysical parsimony. Alternatively, we could piggy-back on the structure of the quantities: apply the scalar multiplication at the representational level and find out which new magnitudes are now represented by the quantity that represented the old magnitudes. Dasgupta develops a mass-counterpart theory that might be used for this purpose [3] (although this basically boils down to a passive Leibniz Scaling, as defined below). If one feels that either of these options would be cheating, it also suffices for the purposes of this paper to consider any automorphism that is not the identity.
} 
masses and mass relations is to be understood as being between magnitudes and relations, not quantities and relations. To every metaphysically possible world corresponding to a structure of mass relations, one would expect ${ }^{39}$ there to correspond (via active Leibniz scalings) uncountably many possible 'absolutist worlds' with different absolute masses (understood as magnitudes) that are all nevertheless compatible with the mass structure in the corresponding 'comparativist world'. (In turn, to each of these absolutist worlds there correspond (via passive Leibniz scalings, see below) uncountably many representationally distinct possible worlds (also known as models), all differing in the quantities assigned to the absolute masses, but we are not interested in those sets of models.) Comparativism should provide at least one metaphysically distinct (and dynamically allowed) possible world for each empirically distinct possible world allowed by absolutism. If the metaphysically distinct worlds that comparativism acknowledges fail to differentiate between those distinct empirical possibilities, then comparativism is wrong. If, on the other hand, the set of all the metaphysically distinct possible worlds acknowledged and dynamically allowed by comparativism contains all the empirically distinct possible worlds (that are dynamically allowed by absolutism), then we may opt for comparativism over absolutism based on an Occamist norm.

Interestingly, whereas in the substantivalism-relationalism debate it is generally accepted ${ }^{40}$ that the Static and Kinematic Shift provide, if anything, a challenge for the substantivalist, and the Rotational Dynamic Shift a challenge for the relationalist, in the mass debate this is not at all the case. Comparativists believe that a Leibniz scaling has no empirical effect (i.e. Leibniz Scalings are a symmetry/absolute masses are undetectable/Leibniz Scalings relate empirically indistinct worlds) and thereby proves their view correct, whereas absolutists claim that it does have empirical consequences and thus proves their view to be correct.

It is important to note that all three approaches centre around an active version of the Leibniz scaling transformation. That is, we ask what happens when we change something out there, that is, what happens when we change the magnitudes, which are part of the metaphysics. Thus, we are not asking what happens if we instead apply the following transformation at the level of representation:

Passive Leibniz (Mass) Scaling: ${ }^{41}$ A uniform scalar multiplication of each of the quantities representing the absolute mass magnitudes of all the particles, ceteris paribus (i.e. whilst keeping everything that is represented, including those magnitudes, unchanged).

This is just a change of unit. It is obvious that a change of unit, like a change of coordinates in the space case, does not change anything in the real world (assuming we consistently change all the other units, say of Newton's constant, as well). It is just a human choice of applying different labels.

We may wonder whether these three approaches should be considered as equivalent approaches to the active Leibniz Scaling argument against/in favour of the empirical adequacy of comparativism, or whether they constitute three separate arguments, potentially even with mutually inconsistent conclusions. In the space debate the three approaches seem to be used interchangeably - except perhaps in the context of the Static Shift.

The notion of symmetries of physical theories and a justification of their associated symmetryto-(un)reality inference are notorious topics in the recent philosophy of physics literature, see

\footnotetext{
${ }^{39}$ Except, in a sense, in the case of regularity comparativism [6].

${ }^{40}$ Despite, ironically, Clarke having been the one to introduce the Static Shift $[54,55]$.

${ }^{41}$ This is closely related to Dasgupta's mass-counterpart theory [3].
} 
for instance Dasgupta [56] and references therein, as well as Møller-Nielsen \& Read [57]. Instead, I will focus on the undetectability approach, including the corresponding version of the Occamist norm, to be developed and criticised below. I will argue that, to the extent that it is distinct from the possibility checking approach, it is wrong. To the extent that it is equivalent to the possibility checking approach, I suggest that we simply move to that more perspicuous approach, as I have done elsewhere [22, Ch.3] [35].

\section{Dasgupta's Comparativism}

\subsection{Dasgupta's Razor}

Dasgupta, in Symmetry as an Epistemic Notion (Twice Over) [56], considers several reasons one might have for believing that features such as absolute masses are redundant (over and above mass ratios), and ultimately settles on an epistemic notion of redundancy, that is undetectability. After first discussing non-epistemic inferences to the unreality of a feature based on that feature either being subjective, or being redundant in the sense of making no difference, or being arbitrary, or being explanatorily dispensable, etc., he concludes that none of these inferences works. Dasgupta points out that we should never have expected these non-epistemic approaches to work in the first place:

If we could see or detect [this feature, eg. absolute mass], we should believe that it is real regardless of whether it is redundant in any of the above senses. So a necessary condition on our reasonably believing that it is unreal is that we do not believe that it is detectable. And from there one might argue that a necessary condition on our reasonably believing that it is unreal is that we believe that it is undetectable. If that is right then it is hard to see how any non-epistemic reconstruction of the inference in terms of redundancy could work. [56, p.850]

In Absolutism vs Comparativism about Quantity [3], Dasgupta applies this reasoning to the absolutism-comparativism debate. From both papers, we may reconstruct his undetectability approach - to which he explicitly commits, despite often sliding between talk about undetectability and about possibility checking - to the general schema for the comparativist argument, Dasgupta's Razor:

$\mathbf{P}_{\text {det }} \quad$ Empirical adequacy: Absolute masses are undetectable (over and above the mass ratios).

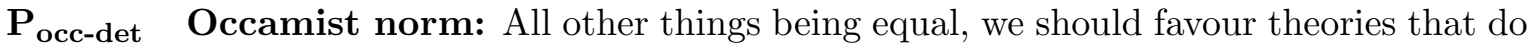
not posit undetectable structure.

$\mathbf{C}_{\mathbf{c}} \quad$ The theory without undetectable absolute masses (i.e. metaphysical comparativism) is favoured over the theory that does posit them (i.e. metaphysical absolutism).

Dasgupta's undetectability approach skips the link with metaphysical parsimony, and homes in directly on undetectability as an epistemic vice. This would circumvent my criticisms against the metaphysical parsimony of comparativism brought forward elsewhere [35]. But it is not directly clear that a theory that posits a feature that, despite being undetectable, provides an 
enormous overall gain in metaphysical parsimony or simplicity, should be disfavoured. Why should the 'all other things being equal' clause not also range over metaphysical parsimony and simplicity? In other words, if the Occamist norm is explicated directly in terms of undetectability rather than metaphysical parsimony, we would expect an additional 'metaphysical parsimony adequacy' premise. In fact, Dasgupta acknowledges ${ }^{42}$ as much:

It remains possible that dispensing with the feature yields a theory that has too many other vices to warrant belief, such as being too inelegant or complex. In that case, we would have empirical evidence of sorts that the feature is real, in the sense that our all-things-considered best empirically confirmed theory implies that it is real. But that is a situation in which all else is not equal. [56, p.854]

In other words, even if Dasgupta's first quote in this section is right about the undetectability of a feature such as absolute mass being a necessary condition for inference to the nonexistence of that feature, it does not follow that undetectability is a sufficient condition.

However, I will not dwell on the validity of Dasgupta's razor, since 1) one of my aims is to argue that the first premise is, in some sense, false, 2) another aim is to point out that it is wrong to equate comparativism with the view that necessarily denies that absolute masses are detectable in that sense, and 3) the most important message is that the notion of undetectability is ill-suited in the first place.

\subsection{Dasgupta's Comparativist Law}

Since we are evaluating the truth of dynamic comparativism, we need to first say something more about the dynamics, that is Dasgupta's comparativist interpretation of Newton's three laws and the Law of Universal Gravitation. Newton's first and third laws are the same in the absolutist and comparativist framework. The second law is standardly written in the following symbolic notation:

$$
f_{q}=m_{q} \cdot a_{q},
$$

where I have added the subscripts to indicate that this formula is standardly interpreted as relating the quantities $f$ (force), $m$ (mass) and $a$ (acceleration).

How to interpret this law? On one interpretation this law governs absolute magnitudes of mass, acceleration ${ }^{43}$ and force:

(L1) For any material thing $x$,

(a) For any reals $r_{1}$ and $r_{2}$, if $x$ has mass $r_{1} M^{[44]}$ and acceleration $r_{2} A$, then $x$ has force $r_{1} r_{2} F$ acting on it.

(b) For any real $r_{3}$, if $x$ has force $r_{3} F$ acting on it, then there are reals $r_{4}$ and $r_{5}$ whose product is $r_{3}$, such that $x$ has mass $r_{4} M$ and acceleration $r_{5} A$. [3, p.130]

\footnotetext{
${ }^{42}$ See also [3, p.133-4].

${ }^{43}$ Although I have taken the qualifier 'absolute' in 'absolute masses' to mean 'monadic' (cf. fn.4), for an absolutist about mass these masses are also intrinsic, even though they will not necessarily be for a comparativist who is a realist about absolute masses (eg. regularity comparativism [6]). In the case of acceleration magnitudes, however, I take it that even an absolutist about those magnitudes will not (necessarily) hold that they are intrinsic, since, according to the "at-at" theory, acceleration is reducible to the limit of velocity differences (which in turn are reducible to a limit of differences in position), cf. $\S 8.3$.

${ }^{44}$ See fn.38.
} 
Dasgupta argues that if L1 is the correct interpretation of the law, then under a Leibniz Mass Scaling - leaving the forces and the accelerations unchanged - the law will not obtain anymore [3]. This should be surprising to the comparativist, since such a Leibniz scaling is equivalent to the identity operation within their framework, and should thus not change whether the law obtains - that is, it should not turn a solution into a non-solution. Under this interpretation, the truth of this law then depends on absolute masses over and above mass ratios. Dasgupta therefore argues that L0 should be interpreted instead as governing only magnitude relations:

(L2) For any material things $x$ and $y$ [in the same world],

(a) For any reals $r_{1}$ and $r_{2}$, if $x$ is $r_{1}$ times as massive as $y$ and is accelerating at $r_{2}$ times the rate of $y$, then $x$ has $r_{1} r_{2}$ times as much force acting on it as $y$. (b) For any real $r_{3}$, if $x$ has $r_{3}$ times as much force acting on it than $y$, then there are reals $r_{4}$ and $r_{5}$ whose product is $r_{3}$, and such that $x$ is $r_{4}$ times as massive as $y$ and is accelerating $r_{5}$ times the rate of $y$. [3, p.130-1]

If this law obtains in one world, then a Leibniz scaling creates a world in which this law also obtains.

Dasgupta justifies this move by claiming that there is no empirical evidence that would confirm L1 over his interpretation of the law L2. This justification is of no use, since it is circular. We are looking for a formulation of the laws of NG such that they render the absolute masses empirically redundant, that is undetectable. We thus can not assume from the start that choosing this law would be empirically unproblematic. What seems to me to be the implicit but incorrect justification behind the alternative interpretation of the law, luring authors such as Dasgupta mistakenly towards comparativism, is kinematic comparativism and the resulting many-to-one relation between quantities and the magnitudes they represent. We will, for now, acquiesce and discuss Dasgupta's analysis of the threat from Leibniz scaling via his interpretation of the law.

Dasgupta does not discuss the actual force law that governs the determinable that we are focusing on: mass. Presumably Newton's law of gravitation could be similarly (re)interpreted $[22, \S 2.2]^{45}$. We will return to this surprising omission below.

\section{Dasgupta: Absolute Masses are Undetectable}

In this section I present (my interpretation of) Dasgupta's set-up and analysis of ways in which a Leibniz Mass Scaling may seem to threaten the empirical adequacy of comparativism. Apart from some critical comments along the way, my own analysis will not be presented until the subsequent section, where I argue that both Dasgupta's set-up and his analysis of the scenarios are mistaken.

\footnotetext{
${ }^{45}$ It is not clear though that either this reinterpretation of the gravitational law, or L2, really are laws. They are not in the form of differential equations, and thereby not suitable for solving initial value problems. Moreover, it can be shown that highly deviant evolutions satisfy these two 'laws' [22, §4.4.1]; solutions that we would expect not to be physically allowed by Newtonian Gravity. Machian comparativism overcomes both these problems [35] [22, §4.2.3].
} 


\subsection{Intra-world Leibniz Scaling}

Consider a Newtonian world W with two spatially separated subsystems S and $\mathrm{S}^{*}$ which differ by a Leibniz Mass Scaling. As Dasgupta interprets this, $\mathrm{S}$ and $\mathrm{S}^{*}$ are copies of each other insofar as the interparticle distances and the velocities of and forces on each particle in $\mathrm{S}$ are the same as their counterpart in $\mathrm{S}^{*}$, but all the masses of the particles in $\mathrm{S}^{*}$ are, say, twice that of their counterparts in S. An example is shown at the top of Figure 1. Applying L1 to each of the subsystems separately and then comparing the results tells us that the particles in $\mathrm{S}^{*}$ will accelerate at half the rate of their counterparts in $\mathrm{S}$.

To the comparativist about mass this scenario looks different. Not only are the force ratios and distance ratios in $\mathrm{S}$ identical to their respective counterparts in $\mathrm{S}^{*}$, but also the mass relations. This is depicted in subfigure (b) of Figure 1. Can the comparativist account for the differences in accelerations? Applying L2 to each subsystem separately seems to fail to provide those different accelerations since everything that is governed by L2 (i.e. force relations, mass relations and acceleration relations) is identical between both systems.

One may respond that it is no problem that the acceleration relations within $\mathrm{S}^{*}$ are the same as their counterpart relations in S. In fact, the absolutist picture tells us they should be: the absolute ${ }^{46}$ acceleration of each particle in $\mathrm{S}^{*}$ is half that of the acceleration of its counterpart in $\mathrm{S}$, which leaves all the intra-system acceleration relations invariant. However, the issue was never that the intra-system relations cannot be accounted for, but the inter-system acceleration relations. In other words, the comparativist is not required to account for different accelerations nor acceleration relations within each system, but a difference in accelerations between both systems, or so Dasgupta claims.

This may seem to indicate that absolute masses are not redundant: they are necessary to account for the empirical data, in this case inter-system acceleration relations. However, the analysis above makes the mistake of only applying L2 to each subsystem separately. We are tempted to do so since in the absolutist framework all the relevant information is stored locally within the subsystem, namely on each particle. Hence, applying the relevant law (i.e. L1) to each subsystem separately gives us all the relevant absolute magnitudes (i.e. accelerations) for each separate subsystem, and a fortiori all the relevant magnitude ratios between the two systems. In the comparativist framework not all the information is stored locally: the magnitude ratios between particles in $\mathrm{S}$ and the magnitude ratios between particles in $\mathrm{S}^{*}$ do not merely fail to determine the inter-system relations, they do not constrain them at all. Only when we take into account the inter-system mass relations do we have all the relevant information. Subfigure (c) in Figure 1 depicts the inter-system relations corresponding to the absolutist scenario in subfigure (a). Once we take into account that the inter-system mass relations are such that each particle in $\mathrm{S}^{*}$ is twice as massive as its counterpart in $\mathrm{S}$, that is by applying L2 to the whole world, we have no problem recovering the inter-system acceleration relations. And that is all the comparativist about mass needs to explain, or so Dasgupta claims; no further absolute accelerations (or anything else) are to be accounted for. Getting the acceleration ratios right implies that absolute masses are undetectable. It is not directly clear why it would be true though that no further absolute accelerations (or anything else) are to be accounted for. Comparativism about mass does not by itself imply comparativism about accelerations. Presumably Dasgupta is implicitly invoking kinematic comparativism about accelerations. Since absolute accelerations cannot be expressed directly, but only via comparisons, acceleration ratios are the only empirical data

\footnotetext{
${ }^{46}$ As opposed to the comparative acceleration, rather than the relative acceleration.
} 


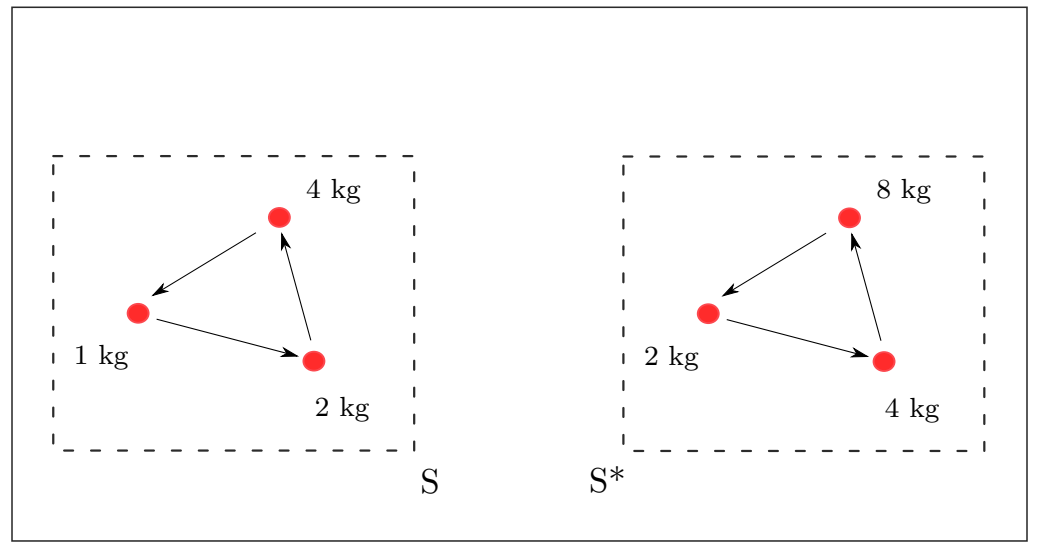

W

(a)

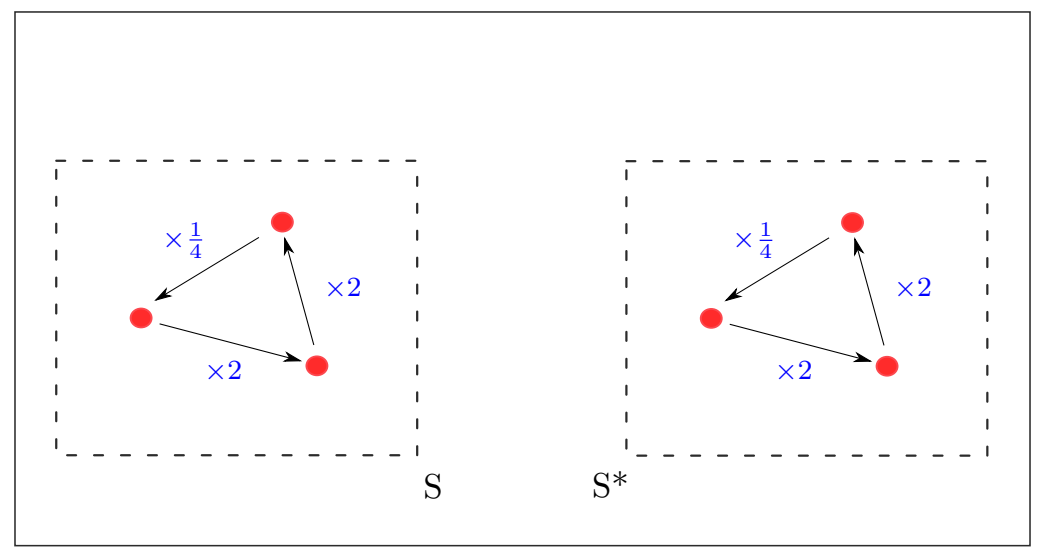

$\mathrm{W}$

(b)



W

(c)

Figure 1: Example of an intra-world Leibniz scaling scenario. An arrow with for instance the label ' $\times 2$ ' represents the following mass ratio: the object at which the arrow points is twice as massive as the object at which the arrow starts. The force on each particle in system $\mathrm{S}$ is, ex hypothesi, identical to the force on its counterpart in $\mathrm{S}^{*}$; they are omitted from this picture. 
that is to be accounted for.

\subsection{Inter-world Leibniz Scaling}

In the second scenario, the two systems under consideration are not part of the same world W, but are each complete and distinct possible worlds: $\mathrm{S} \equiv \mathrm{W}, \mathrm{S}^{*} \equiv \mathrm{W}^{*}$. They are related via an inter-world Leibniz Mass Scaling. In Dasgupta's words, the objection asks us to consider a possible world $\mathrm{W}^{*}$ in which all absolute masses are uniformly doubled compared to the first world $\mathrm{W}$, whilst the forces, distances and velocities remain the same. Subfigure (a) of Figure 2 is an attempt to depict this scenario, but it is important to realise that certain difficulties arise when representing such a scenario due to the one-to-many relation between magnitudes and the quantities used to represent those magnitudes. The particle that is labelled ' $1 \mathrm{~kg}$ ' in $\mathrm{W}$ has a counterpart labelled ' $2 \mathrm{~kg}$ ' in $\mathrm{W}^{*}$. This is to represent that the mass magnitude of the counterpart in $\mathrm{W}^{*}$ is doubled compared to the original particle in W. However, 'kg' usually obtains its meaning - within a world! - by choosing one privileged particle - within that world - that is represented by the quantity 1 , or $1 \mathrm{~kg}$. The masses of the other particles are then represented comparatively, by expressing them in multitudes of this conventional $\mathrm{kg}$. In the actual world that privileged object is a platinum alloy cube in Paris. The $1 \mathrm{~kg}$ particle $\mathrm{W}$ is the analogue of the cube in Paris. In $\mathrm{W}^{*}$ the magnitude of the counterpart of the 'cube in Paris' is doubled compared to the original. However, if ' $\mathrm{kg}$ ' is supposed to label the standard mass in Paris, then the counterpart in $\mathrm{W}^{*}$ should, by definition, also be labelled ' $1 \mathrm{~kg}$ ', even though it would refer to a magnitude twice as large as the magnitude refered to by ' $1 \mathrm{~kg}$ ' in W. This would of course completely hide exactly what the figure is supposed to convey. Hence, usage of ' $\mathrm{x} \mathrm{kg}^{\prime}$ ' in $\mathrm{W}^{*}$ is to be understood as representing the magnitude that would be represented by that label in W.

Once again, we consider whether Dasgupta's comparativism gets the empirical data right, in accordance with the first premise of Dasgupta's razor. As Dasgupta interprets the explanandum raised by this objection: in $\mathrm{W}^{*}$ the masses are uniformly doubled compared to $\mathrm{W}$, so the accelerations should be uniformly halved. At this point one might worry (or rejoice) that this scenario indeed seems to be a much stronger threat to the empirical adequacy than the first. There the explanandum was explained by an inter-system application of L2, that is via intersystem mass relations. Here the systems are worlds, rendering the analogue of the explanans fundamental inter-world mass relations, something Dasgupta is reluctant to commit to [3, p.115]. There is no arrow in Figure 2 that would serve as the analogue to the inter-system arrow that appears in subfigure (c) of Figure 1. The other side of this coin is however that there is in fact also no inter-world explanandum (in Dasgupta's sense). There are no fundamental interworld acceleration relations, nor are there any emergent or epistemically accessible inter-world relations that need to be explained. We have no means of magically travelling to other possible worlds with our rod and clock to see how the accelerations there relate to our actual world. Applying L2 to the mass relations in $\mathrm{W}$ gives the correct acceleration relations in $\mathrm{W}$; applying $\mathrm{L} 2$ to the mass relations in $\mathrm{W}^{*}$ gives the correct accelerations in $\mathrm{W}^{*}$ (namely the same as in $\mathrm{W}$ ). And this is all that needs explaining. Or so it seems when the explanandum is explicated in the way Dasgupta does. 


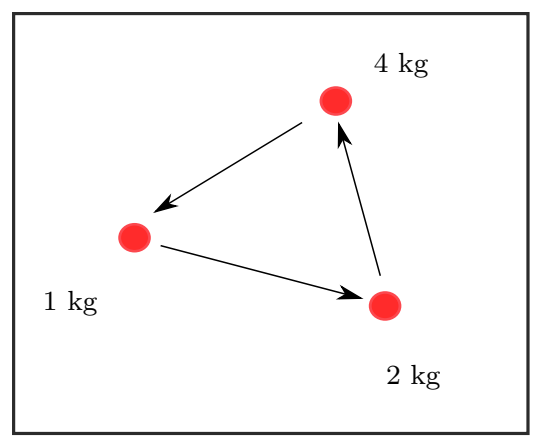

W

(a)

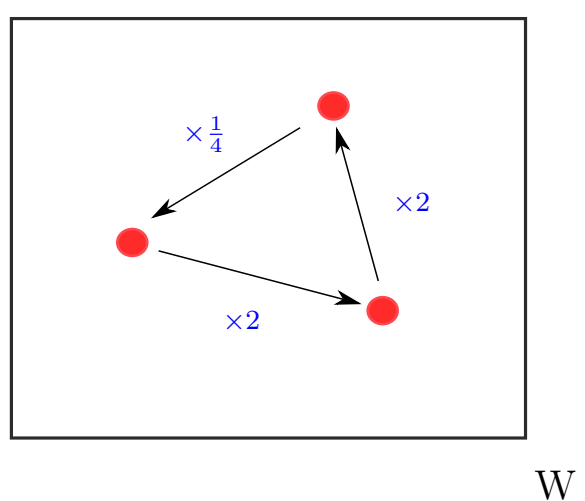

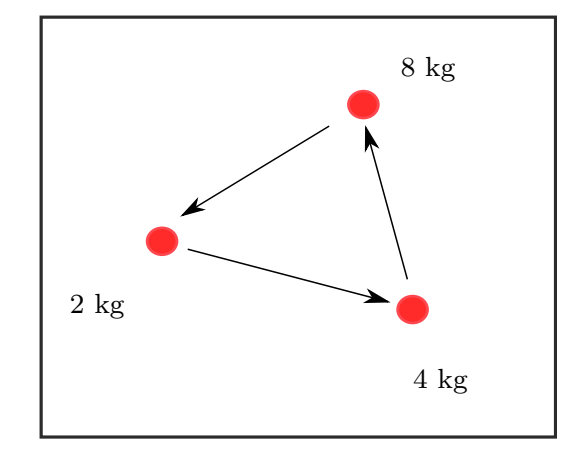

$\mathrm{W}^{*}$

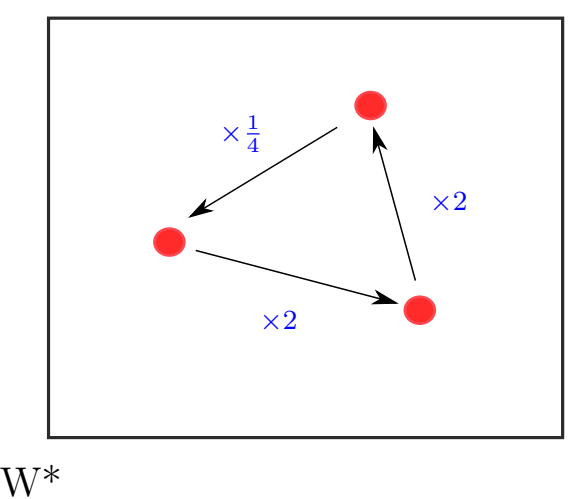

(b)

Figure 2: Example of an inter-world Leibniz scaling scenario. Subfigure (a) represents the scenario in absolutist terms. For the usuage of the label ' $\mathrm{kg}$ ' in $\mathrm{W}^{*}$, see the comments in the text. Subfigure (b) represents the information about mass available to the comparativist about mass. 


\section{Response to Dasgupta}

The previous scenarios purported to show, supposedly unsuccessfully, that two versions of Leibniz Scalings both lead to detectable differences, which according to Dasgupta is to be equated with the detectability of absolute masses, which in turn is to be equated with the empirical inadequacy of comparativism. In the intra-world scenario Dasgupta explicates this explanandum of 'getting the empirical data right' as explaining the acceleration relations, specifically the inter-system accelerations relations. In this scenario he applies L2 to both systems as a whole - in particular the inter-system mass ratios - to explain this explanandum; in the interworld scenario there is in fact no such explanandum. It seems that Dasgupta's comparativist can account for all the relevant empirical data, which supposedly renders the absolute masses undetectable.

It is in formulating the explananda that the first of two crucial flaws appears in Dasgupta's analysis of and response to both scenarios. Observables in Newtonian Gravity are the trajectories of particles and the relations between them. Those follow, in the case of absolutism, from initial conditions plus the laws of the theory (i.e. L1 plus the gravitational law). All Dasgupta has done is check whether (his interpretation of) the 'law' (L2) obtains in all scenarios. L2 governs mass relations, force relations and acceleration relations. (We will get to the forces in a second.) It is indeed the case that the acceleration relations in each system, and between each system, are correctly predicted by L2 for each scenario. Checking only whether the correct acceleration relations obtain in no way ensures that uniform mass doubling does not change the particle trajectories and the relations between those trajectories. These might depend on more than merely acceleration relations, and as I will show below they in fact do. If L2 (presumably together with the comparativist version of the gravitational law), fails to provide those trajectories, we should question whether they even deserve to be called laws (see fn.45), or are merely regularity statements that happen to be true of both scenarios.

The second flaw sneaks in right at the beginning, when the scenarios are described. We are asked to imagine that only the masses are doubled, and that everything else remains the same, in particular the forces. The ceteris paribus clause in the Leibniz Mass Scaling is misinterpreted to include forces ${ }^{47}$. In other words, the force magnitude (or magnitude relation) mentioned in Newton's Second Law is viewed as an independent variable, whereas in fact it represents a 'slot' which needs to be filled by another specific force law. Forces do not 'float free', but have sources, and in Newtonian Gravity the source of the only force available is mass, exactly the thing that we are asked to vary in these scenarios. Dasgupta does acknowledge that he is ignoring this issue (footnotes 27 and 30), but it is wholly unclear why this would be justified. Indeed, on reflection it might strike one as rather strange that the gravitational force has not been mentioned a single time in our lengthy discussion of the detectability of mass. Of course we could introduce, for instance, electromagnetic forces, which would indeed remain invariant under uniform mass doubling. However, this does not mean that the gravitational force suddenly disappears. Although for some choices of parameters the gravitational force becomes negligible and the forces involved seem independent of mass for all practical purposes, this would not hold anymore once we start scaling the masses uniformly by large scalars. Moreover, including an electromagnetic force means extending Newtonian Gravity, whereas here we are concerned with standard NG. (Admittedly, Dasgupta seems to be interested in Newtonian Mechanics more broadly, but 1) no realistic approximation of the actual world could leave out the gravitational

\footnotetext{
${ }^{47}$ I believe Baker's Friction World and Shell World arguments to suffer from the same mistake [21, 58].
} 
force ${ }^{48}$, and 2) considering Newtonian Mechanics more broadly does not mean considering only Newton's three laws. Newton's three laws are by themselves not a theory: the F-slot needs to be filled by something in order for it to be a well-defined theory. Forces require a source. ${ }^{49}$ ).

Not only does Dasgupta analyse the objections incorrectly by explicating the explanandum incorrectly, but the scenarios he proposes are inconsistent in the first place - he misinterprets the ceteris paribus clause in the Leibniz Scaling transformation. In the following section I will first provide the correct analysis within the inconsistent scenario. Even then the absolute masses turn out to be empirically meaningful (a corollary of which is that they would be empirically meaningful as well in an 'NG plus dominant electromagnetic force' theory). Perhaps two wrongs make a right: perhaps the dependence of the force on the mass can compensate for the empirical difference arising directly from the mass doubling. In the subsequent section I give the fullfledged analysis including gravitation. Only a force law that is $\propto m^{1}$ would right the wrong; with a gravitational force law that varies as $\propto m^{2}$, a uniform mass doubling, properly interpreted, leads once again to a detectable difference.

\subsection{Detectability: Dependence of Trajectories on Absolute Masses}

In this section I show an example of a set of trajectories of massive particles that is detectably different when we uniformly vary the masses of those particles (while keeping the forces constant). (And if the two systems under consideration are two distinct possible worlds, there are no inter-system mass ratios that could be used in any way as an explanans.)

Trajectories are collections of spatial points labelled with a time parameter. As such, a substantivalist framework might seem best to formulate the following example. However, 1) I want the example to convince relationalists as well, and 2) there being substantival space and time does not by itself give us epistemic access to length and duration. We could use some extra particles to serve as a rod and a clock. Imagine a world with two force-free particles, subfigure (a) of Figure 3. Without loss of generality I will assume that, within a substantival framework, they are both at rest. The distance between these two particles, 'the rod', serves as the standard for the length unit $d$. This allows us to introduce a third force-free particle which, in substantival terms, is moving away from the rod with a non-zero constant velocity (subfigure (c)), and thereby serves as a clock. The unit of time $t$ is defined as the time it takes the third particle to move a distance $d$. We now add a fourth particle, to which a force is applied (subfigure (d)). Applying L1 ${ }^{50}$ gives us its trajectory. If the masses would be uniformly doubled whilst keeping the distances, velocities and forces the same (subfigure (e)) - that is, we are considering a specific instance of the inter-world Leibniz Mass scaling scenario-L2 would not recognise the difference; the force relations and mass relations do not change, and therefore neither do the acceleration relations. However, the absolute acceleration - as opposed to comparative rather than relative acceleration - does change. Given that the initial velocities of the particles do not change, the trajectories of the first three particles do not change since they are force-free, but the trajectory of the fourth particle changes due to its change in absolute acceleration. Using our rod and clocks as standards for length and time, we measure a difference in (relative) trajectories that even the comparativist must acknowledge. Absolute masses are empirically meaningful!

\footnotetext{
${ }^{48}$ Unless it is being reduced to, say, the curvature of spacetime, of course.

${ }^{49}$ I believe that both these problems also (fatally) plague Baker's Friction World argument $[21,58]$.

${ }^{50}$ Technically L2 could not even be applied here, since there are no non-zero force relations. This could be remedied by introducing another particle with a force acting on it.
} 


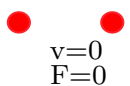

(a) The rod

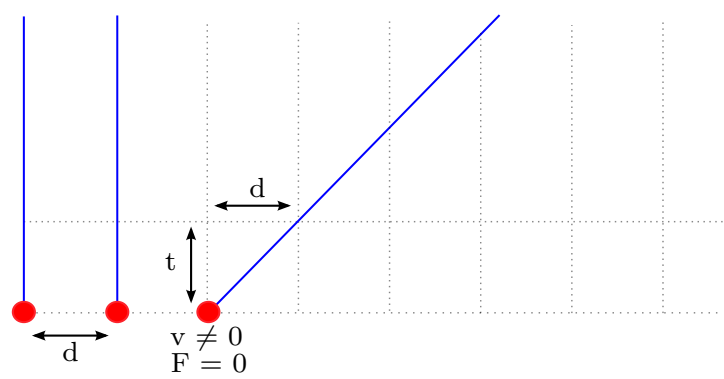

(c) The clock

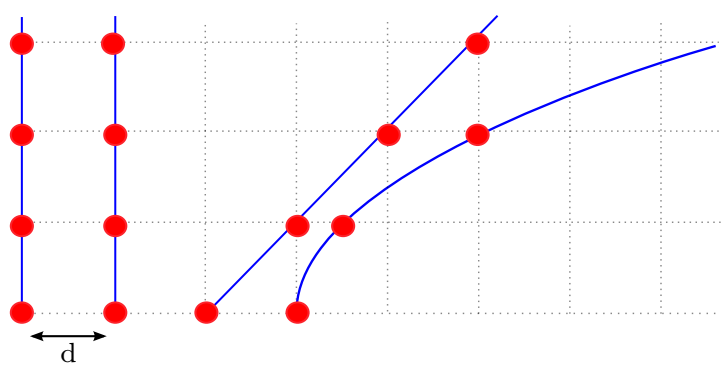

(e) Uniform mass doubling

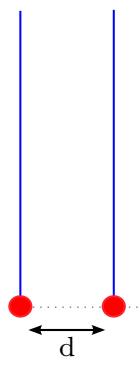

(b) The rod

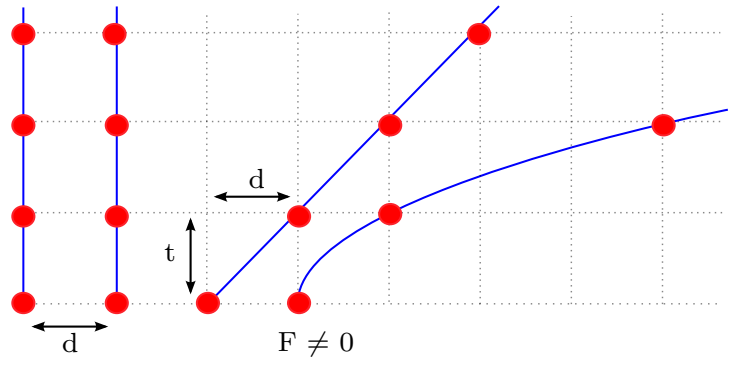

(d) Accelerating particle

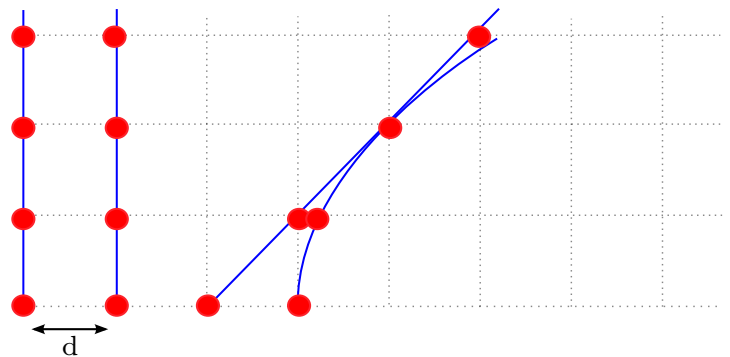

(f) Uniform mass quadrupling

Figure 3: Example of particle trajectories detectably changing under uniform mass doubling. The red dots are massive particles; the blue lines their world lines. The two force-free particles in (a) and (b) serve as a rod; with this standard for length a third force-free particle, (c), can be used to function as a clock. 
Dasgupta's analysis only works in situations with for instance two particles starting at the same location whilst being at rest. In that case $\frac{s_{1}}{s_{2}} \propto \frac{a_{1}}{a_{2}} \propto \frac{m_{1}}{m_{2}}$, where $s$ is the distance traversed. Transformations that leave the mass relations invariant are then not detectable. In general however we need to take into account the initial locations and velocity:

$$
\frac{s_{1}}{s_{2}}=\frac{x_{1}+v_{1} t+\frac{1}{2} a_{1} t^{2}}{x_{2}+v_{2} t+\frac{1}{2} a_{2} t^{2}}=\frac{x_{1}+v_{1} t+\frac{1}{2} \frac{F_{1}}{m_{1}} t^{2}}{x_{2}+v_{2} t+\frac{1}{2} \frac{F_{2}}{m_{2}} t^{2}} \not \propto \frac{m_{1}}{m_{2}}
$$

Mass only appears in front of one term in each of the numerator and denominator; a Leibniz Scaling will not leave the relative trajectories invariant.

I introduced a rod and clock to be able to operationally differentiate the different scenarios resulting from a Leibniz Scaling. If we had a universe with only, say, the third and the fourth particle, epistemologically speaking the bets would be off. In such a universe any coordinate system could be chosen that has the same ordering of coordinates as the (imagined) substantival coordinate system - that is any coordinate system in which the particles do not intersect. We might for instance choose a coordinate system in which both trajectories are rectilinear [59]. However, if we would uniformly quadruple the masses in our previous example (subfigure (f)), two of the trajectories coincide at some point in time. Multiplying the masses even further would result in the trajectory of the fourth particle intersecting the trajectory of the third to 'reach its other side', followed by a later intersection to 'return to its own side'. Such intersections of trajectories are recognisable for both the absolutist and comparativist, without even the need for rods and clocks. Coincidence or no coincidence is the crucial empirical difference. This topological criterion $^{51}$ is an empirical feature that both the absolutist and the comparativist can agree on without having to invoke rods and clocks. This is fortunate since we will not be able to construct rods and clocks from force-free particles once we take gravitation into account, as every massive particle will feel a force from the other massive particles.

\subsection{Adding Gravitation: the Comparativist's Bucket}

Let us finally use the full power of Newtonian Gravity by adding gravitation. We will consider yet another, even simpler instance of the inter-world Leibniz Mass Scaling scenario - a simplification of Baker's Earth-Pandora scenario [21] - so that there are no inter-system mass ratios available anyway that could potentially be used as a comparativist explanans. Consider a world, governed by the laws of Newtonian Gravity, with two equally massive particles a distance $r$ apart, each initially moving away from the other with velocity $v$ (such that there is zero angular momentum). What will happen? Whereas this description corresponds to a unique choice of initial variables and parameters for the comparativist, the absolutist will demand that more information is needed: this description is compatible with continuum infinitely many absolute masses (i.e. absolutist worlds differing by a Leibniz Mass Scaling). And, she claims, this choice is important, because it determines whether the gravitational force provides enough of a 'brake on the velocity' to make the two particles end up coinciding. Using L1 and Newton's law of gravitation we can calculate the escape velocity:

$$
v_{e, 1}=\sqrt{\frac{2 G m_{2}}{r}} .
$$

\footnotetext{
${ }^{51}$ See also Schlick's 'method of coincidences' [60-63].
} 
If the initial velocity is larger than the escape velocity the particles will escape, otherwise they will coincide. It is crucial to notice that the escape velocity depends only on the absolute mass of each of the particles, not the mass ratio between the two particles! Hence, if the absolute masses of the particles in our scenario are such that the particles barely escape each other, they will 'collide' (i.e. coincide) in another world which differs only by uniform mass doubling (properly interpreted). The comparativist cannot distinguish these two worlds as far as the initial conditions are concerned, as these - the mass relations, distance relations, velocity relations and the force relations (and therefore also the acceleration relations that follow dynamically from these initial conditions, which are the only things Dasgupta cared about) - are identical. In the comparativist framework this scenario is indeterministic: the initial comparativistic conditions do not predict whether the particle trajectories will intersect or not, an observable difference that even the comparativist recognises. The absolutist has no problem predicting what happens. That we do not have a clock or rod available is no problem, since in one world the particles will never collide, even if one waits infinitely long, while in the other world the particles will collide within finite time. Absolute masses are empirically meaningful! ${ }^{52}$

Newton's bucket similarly shows that the relationalist initial conditions, in particular relative accelerations, are insufficient for an empirically deterministic evolution of the system. Absolute $^{53}$ (rotational) accelerations are empirically meaningful. By analogy ${ }^{54}$, we will call this specific two-particle instance of the inter-world Leibniz Mass Scaling the comparativist's bucket - although it is important to realise that the same issue arises for any other pair of worlds with any number of particles ${ }^{55}$; it is just that the two-particle scenario is the simplest scenario for my purpose as it allows for analytic solutions. Absolute masses are empirically meaningful. (In terms of the symmetry approach: a Leibniz Mass Scaling, interpreted as keeping the distances and velocities the same, is not a symmetry of the theory.)

It is interesting to note that it is not the case that any dependence of the force on mass would produce this problem for the comparativist. If the force would for instance be $F_{g^{\prime}}=\frac{G^{\prime} m_{2}}{r^{2}}$ or $F_{g^{\prime \prime}}=\frac{G^{\prime \prime} m_{1}}{r^{2}}$, the escape velocities would be $v_{e^{\prime}, 1}=\sqrt{\frac{G^{\prime} m_{2}}{r m_{1}}}$ and $v_{e^{\prime \prime}, 1}=\sqrt{\frac{G^{\prime \prime}}{r}}$ respectively. These escape velocities are invariant under uniform mass scaling. These forces would thus cancel out exactly the effect of the mass doubling on the trajectories discussed in the previous section. This result is in sharp contrast with the naive argument considered in $\S 4$ - that dynamic comparativism is logically implied by kinematic comparativism - which would have proven comparativism true a priori. Such a result would both be very surprising, and at the same time make the debate much less interesting. We have now seen that the empirical meaningfulness of absolute masses is truly a non-trivial empirical (i.e. a posteriori) question, although that should

\footnotetext{
${ }^{52}$ In fact, if we place particle detectors infinitely far to the 'left' and 'right', we have in effect constructed the absolute mass detector of which Dasgupta argued it could not exist [3, §8.3].

${ }^{53}$ As opposed to relational.

54 An even closer analogy, using particles rather than a bucket, would be Skow's two-particle scenarios [64] (discussed by Pooley [65]) or Barbour's three-particle scenarios [49, Fig.13]. As all these scenarios are used to make the same point, and Newton's bucket is the most famous of the three, I have chosen to adopt the name 'comparativist's bucket'.

${ }^{55}$ However, in the general multi-dimensional, multi-particle case, presumably only a subset of the space of solutions of measure zero will have colliding particles at all. Perhaps this is even a reason to believe that we can and should ignore that subset. In the general scenario it will then be necessary to make our criterion of empirical equivalence of worlds more strict by considering not only coincidence of trajectories but also angles (i.e. shapes) or ratios of distance over time. The absolutist and comparativist should still be able to agree on this criterion.
} 
not be too much of a surprise.

\subsection{Baker's Response}

Although the comparativist's bucket experiment derives from Baker, he himself does not conclude from it that masses are empirically meaningful. Using the fact that $a=\frac{G M}{r^{2}}$, he rewrites the escape velocity inequality (Equation 2) as $v>v_{e, 1}=\sqrt{2 a r}$. He then concludes that, since no mass appears in this equation, a Leibniz Mass Scaling does not change the trajectories of the particles. The mistake made is that $a$ is interpreted as an independent variable, which is not the case for the standard ideology of NG in terms of distance, velocity and some notion of mass (i.e. absolute or comparative). Finding a new symbol and substituting this into an equation does of course not mean that we should forget what this symbol meant, and in particular that it depends on $m$. Baker's formulation of the escape velocity only serves to disguise the crucial dependence on mass, not to prove it is not there: $v>\sqrt{2 r a(m)}$.

(One might be tempted to respond by changing the standard ideology by promoting acceleration to a fundamental concept, but in order to do so one has to demote another variable, on pain of overdetermining the dynamics, which may lead to inconsistencies. Here we might anticipate that it is possibly the concept of mass that gets thrown overboard altogether, which may not be in the best interest of the comparativist. We will return to this below.)

Although it is unclear why one would do so at this point in the dialectic, Baker then turns to an analysis of the higher-order spatiotemporal notions, velocity and acceleration. Do instantaneous velocities and accelerations (at the initial time) make sense? An object's velocity at time $t$ is ordinarily defined as a derivative, that is as a limit:

$$
v(t)=\frac{d r}{d t}(t)=\lim _{\Delta t \rightarrow 0} \frac{r(t+\Delta t)-r(t)}{\Delta t}=\frac{r(t+d t)-r(t)}{d t} .
$$

On this view, sometimes called the "at-at" theory ${ }^{56}$, only the mass and distances remain as independent variables; the velocity, rather than being instantaneous, is defined reductively via the change in the distance across an infinitesimal period of time. Acceleration can be reduced analogously. We can now again rewrite the escape velocity inequality:

$$
\left(\frac{d r}{d t}\right)^{2}>2 r \frac{d^{2} r}{d t^{2}}
$$

Baker concludes from this equation that not only a Leibniz Mass Scaling does not change whether the inequality is satisfied, but neither does a Leibniz Length Scaling: multiplying $r$ by a constant $c$ multiplies each side of the equation by $c^{2} .{ }^{57}$

Of course the length dimensions on each side of any physically well-defined (in)equality are the same. But from this basic fact about dimensional analysis nothing follows for the physically non-trivial issue of whether dynamic comparativism holds. I have illustrated this elsewhere for the case of mass and Newton's constant - even though the units of Newton's

\footnotetext{
${ }^{56}$ This idea originates with Aristotle and was developed in the Middle Ages [66-68]. In more modern times it is associated, amongst others, with Russell [25].

${ }^{57}$ It may be suggested that scaling $t$ could still change whether the inequality is satisfied, but it is not clear, on the at-at theory, that $t$ remains as an independent variable alongside distances, once one has reductively defined velocity and acceleration in terms of distances. As Baker puts it: "[w] hether the inequality holds or not is a scale-independent fact about $t$ 's neighborhood" [21, p.20].
} 
constants have a (non-zero) mass dimension, it does not follow that a Leibniz Mass Scaling implies a simultaneous scaling of Newton's constant [35]. Here I discuss two ways of seeing what is problematic specifically about Baker's analysis of Inequality 4. More importantly, we will ultimately see, as anticipated, that his analysis, even if it would have been correct, does not bear upon the issue of comparativism about mass in the first place.

If not length, then there are no independent variables left in Inequality 4 which might influence whether it is satisfied or not. Thus, on Baker's analysis, if the particles escape (collide) in one possible world and hence satisfy (violate) the escape inequality, then the inequality will hold in all ${ }^{58}$ (none of the) dynamically possible worlds: in no dynamically possible worlds do the two particles collide (escape). To the extent that we believe that such collision (escape) is empirically possible, Baker's view fails to recover all physically possible worlds of Newtonian Gravity.

What seems to be the case is that Baker, in effect, has assumed an alternative ideology, i.e. an alternative set of initial conditions, consisting only of interparticle distances, $r_{i j}$, in a sense that suggests that there is only one independent dimensionful variable. If this were true, it would not be surprising that all possible worlds have the same evolution: in order to obtain more than one evolution, we need at least two dimensionful magnitudes that can be compared to each other. What has gone wrong in the analysis of Inequality 4 becomes clear when we return to the reductive definitions of velocity (Equation 3) and of acceleration:

$$
a(t)=\frac{r(t+2 d t)-2 r(t+d t)+r(t)}{d t^{2}} .
$$

We see that the notion of a 'Leibniz Length Scaling' is ill-defined. Which $r$ ? $r(t)$, or $r(t+d t)$ or $r(t+2 d t)$ ? Indeed, if all three are simultaneously (and uniformly) scaled by the same factor, Baker's analysis goes through, but this means that a change in distance is coupled to a change in velocity and in acceleration, and these are supposed to be independent, even on the "at-at" theory. It is unclear why a change in one must be accompanied by a change in the other. A Leibniz Length Scaling of only $r(t)$ but not $r(t+d t)$ and $r(t+2 d t)$ (or equivalently $v$ and $a$ ) does not leave Inequality 4 invariant. Baker's implicit ideology cannot just be a single distance (per pair of particles), $r_{i j}$, but must be several independent distances: $\left\{r(t)_{i j}, r(t+d t)_{i j}, r(t+2 d t)_{i j}\right\}$. Or, equivalently but more perspicuously: instead of a fundamental ideology including mass whether absolute or comparative - he seems to suggest an alternative ideology of distances, velocities and accelerations: $\left\{r_{i j}, v_{i j}, a_{i j}\right\}$.

But then we see, as anticipated, that this approach is a reductionist approach: it attempts to reduce mass to spatiotemporal notions. Whether this alternative ideology still reproduces all the physically possible worlds that we normally associate with Newtonian Gravity is an interesting question in itself-I argue elsewhere that the answer is 'no' [34] $]^{59}$. Regardless, this reductionist approach throws out the massive baby with the bathwater - it throws away fundamental absolute masses not in exchange for fundamental mass ratios but fundamental

\footnotetext{
${ }^{58}$ Strictly speaking this is only true for possible worlds with only two particles, call these the scarce worlds. If there are more particles, for simplicity say several (approximately) isolated two-particle systems, these systems might stand in the correct mass relation to the alpha-two-particle system that is the counterpart of the system in the scarce worlds to allow for a different evolution. The question then remains however how to determine which of the two-particle systems is the alfa-system. These issues are discussed elsewhere [35].

${ }^{59}$ A Machian rather than this Laplacian [34] form of reducing mass to acceleration is discussed elsewhere [6] under the name of 'regularity eliminativism', but leads to a form of weak absolutism, rather than a defence of comparativism.
} 
non-mass magnitudes. This form of reductionism would rule out both strong absolutism and comparativism, but prima facie be compatible with either weak absolutism or comparativism. It goes no way towards defending weak comparativism over weak absolutism. In fact, the comparativist's bucket suggests that it should be supplemented with weak absolutism. The mass ratios are grounded in absolute masses which in turn are grounded in spatiotemporal (i.e. non-mass) magnitudes.

\section{Evaluating the Undetectability Approach}

Now we have realised that the real explanandum is the (relative) particle trajectories (pace Barbour [49]) and not the acceleration ratios, and that those trajectories detectably differ depending on the absolute masses, it seems that we should conclude from the discussion so far that the undetectability approach to the argument for comparativism actually backfires and proves absolutism correct instead. Although this conclusion indeed holds up, for the same reasons, on the possibility counting approach — as shown elsewhere $[22,35]$ — we will now make a U-turn, arguing that the undetectability approach is a bad way of explicating the issue of the empirical adequacy of comparativism in the first place.

There are three reasons why the empirical meaningfulness/ meaninglessness of absolute masses comes apart from their (un)detectability and from the empirical (in)adequacy of comparativism. Firstly, the empirical meaninglessness of absolute masses comes apart from the empirical adequacy of comparativism once we realise that the discussion so far has confused fundamentality and reality. This is not uncommon in the analogous debate about space: relationalism is sometimes portrayed as denying the reality of spacetime ${ }^{60}$, which is false for most relationalists (except perhaps Leibniz himself). The issue at the heart of that debate is whether the spatiotemporal relations between material objects are fundamental, or grounded in the spatiotemporal relations between the spacetime points they occupy. In the former case, spacetime is still a real feature that emerges ${ }^{61}$ from the network of fundamental spatiotemporal relations between matter. Similarly, the comparativist does strictly speaking not deny what we may call realism about absolute masses ${ }^{62}$ :

Realism about Absolute Masses: Absolute masses are empirically meaningful.

Comparativists only deny that absolute masses are more fundamental than mass ratios. Not being fundamental does not imply not being real. A chair is not fundamental, but it is real. An absolutist does not deny the reality of mass ratios, but merely their fundamentality. Similarly, absolute masses could in principle be grounded in mass ratios (and perhaps other non-mass properties). This is admittedly a counter-intuitive position, at least prima facie, and antirealism about absolute masses is certainly very much in the spirit of comparativism which is

\footnotetext{
${ }^{60}$ One may, for instance, have expected this mistake from the title of Pooley's forthcoming book on the substantivalism-relationalism debate, The Reality of Spacetime, although this does not in fact occur in the book [65].

${ }^{61}$ For instance, but not necessarily, as a 'real pattern', a phrase coined by Dennett [69].

${ }^{62}$ In fact, Dasgupta seems to have some sympathy with realism about absolute masses, insofar as he finds it important that comparativism must be able to explain what he calls the kilogram facts (e.g. the fact that my laptop is $2 \mathrm{kgs}$ ), which seem closely related to absolute masses. He even develops a novel, plural notion of grounding $[3,46]$ in order to be able to explain these facts. It is not directly clear to me how he takes this sympathy with realism about absolute masses to be compatible with denying the detectability of absolute masses.
} 
probably the reason why to my knowledge nobody else so far has adopted such a realist comparativist position. Nevertheless, if one agrees with my argument for the empirical meaningfulness of mass in $\S 8.2$, absolute masses are clearly real. This is by itself an important result, even if it does not immediately prove absolutism. In fact, many may find the issue of the realism of absolute masses more interesting than the actual absolutism-comparativism debate as it has been defined in this paper. They may even want to go as far as to redefine absolutism (comparativism) as realism (anti-realism) about absolute masses. ${ }^{63}$ This is however mere semantics, so I have chosen to stick to the standard definitions as they occur in most of the literature on this topic. In light of this argument for realism about absolute masses (i.e. the comparativist's bucket), an obvious loophole ${ }^{64}$ the comparativist might want to exploit is to remain insistent on the non-fundamentality of absolute masses - the letter of comparativism-whilst at the same time accepting their reality-giving up the spirit of comparativism. This option is discussed under the name 'regularity comparativism' elsewhere [6]. Comparativism thus clearly is consistent with absolute masses being empirically meaningful.

The other two reasons are two sides of the same coin. They relate to equating the empirical meaningfulness/meaninglessness of absolute masses with their (un)detectability. In Section 8 we showed that absolute masses are empirically meaningful, because they are correlated with trajectories in an observable way. In other words, they are empirical-difference-makers. Does that mean we have detected/determined/gained knowledge of which absolute mass this object has [imagine me pointing at any of the objects in an instance of the scenarios described in the previous sections]? Can you express to me what the absolute mass - the quiddity - is of this object? ${ }^{65}$ No. Conversely, if absolute masses had not been empirically meaningful, what would it have been exactly that we would have failed to detect?

Consider again sending objects to aliens; this time two $4 \mathrm{~kg}$ gloves. When we ask after their mass ratio and shape, the aliens have no problem determining and then reporting those to us: 1 and 5 (fingers), respectively. But when we ask what the absolute mass is, they can only determine that the existence of the absolute masses (whichever specific absolute masses they are) of the tiles that ground their mass ratios influences the trajectories of those tiles. They cannot express the specific absolute masses (more) directly, that is they cannot expres them beyond that - i.e. beyond the indirect statements 'this is the mass that corresponds to this/ that trajectory' or 'this mass is the same as/ different from that mass' - in contrast to the way in which they can (more) directly state the number of corners. Conversely, if those absolute masses had not made a difference to the trajectories, there was no well-defined question we could ask the alien (about absolute masses) that they would not have the answer to. Asking them what the absolute mass is was not a well-defined question, since it is not clear what could ever count as an answer (other than, eg. 'the same absolute mass as that other object' or 'the mass that corresponds to that trajectory'). We could only ask them if the mass of the tiles is twice as large as it actually is, to which the trivial answer is no, or how the masses compare to those of other objects.

\footnotetext{
${ }^{63}$ Arguably, Roberts' rough definition of comparativism could be interpreted as such: "A comparativist about a quantity-type - about mass, for instance - says that what has significance is relations among the values of quantities of that type, rather than values of particular quantities of that type" (italics in original) [4, p.3] (see also his fn.3).

${ }^{64}$ If one had chosen to redefine absolutism (comparativism) as realism (anti-realism) about absolute masses, this loophole would not be an option. Regularity comparativism would be off the table, ab initio.

${ }^{65}$ Since comparativist mass ratios are generally also quidditistic (§3)-Machian comparativism being the exception [35] - a similar problem seems to occur for comparativist mass ratios.
} 
A parallel to this conundrum is generated by the (supposed) undetectability of absolute positions in NG [55,70,71]. Maudlin contrasts the inexpressibility of our supposed ignorance of absolute positions (which is therefore no true ignorance, since there is no epistemic vice that an Occamist norm $\left(\mathrm{P}_{\text {occ-det }}\right)$ can latch onto) with the expressibility of our actual ignorance about (the direction of) absolute velocities.

The essential difference between the static and kinematic cases lies in the semantical role that indexicals or demonstratives play for terms denoting places as opposed to the roles they play in terms denoting velocities. Since all absolute places are qualitatively identical, the only way we can possibly refer to them is either by direct ostension or by using a definite description which makes reference to some material object [i.e. kinematic relationalism]... We can then formulate meaningful counterfactuals about worlds where everything would be displaced from its actual location, but we can also be assured that they are counterfactuals, that they do not describe the world as it is. ...

We can ... sensibly ask about the Earth's absolute velocity without having, even in principle, a means to determine what it is. But we can only sensibly ask about the position of the Earth by asking for its position relative to some determinate set of coordinates [i.e. kinematic relationalism], and the linguistic wherewithal needed to establish the coordinates also provides us the means of answering the question. In sum, the only way that the static shift can be formulated is something like, "what if God had created the material universe oppositely oriented to the way it is oriented now?", and this is clearly a counterfactual situation. But we can ask "what if God created the material universe at absolute rest?", not knowing whether we describe a counterfactual situation or not. [55, p.190-1, italics in original]

In our case, if absolute masses had turned out to be empirically meaningless, I could not detect/determine and get to know how (absolutely) massive a specific object was, but I could not express that ignorance by stating which specific absolute mass I do not know the object has. For example, 'I cannot know whether the absolute mass is twice the actual mass' is clearly false, as is 'I cannot know, in principle, whether the absolute mass is the same as the absolute mass of that other object'. This failure of expressibility should not surprise $u s^{66}$ : this is the content of kinematic comparativism, this is what it means, by definition, to be a dimensionful magnitude - in fact, as Table 1 shows, typical examples of expressible ignorance and knowledge are all dimensionless notions, and typical examples of inexpressible ignorance and knowledge are all dimensionful notions. This is why the Leibniz Scaling is (necessarily) formulated 'comparatively', as a multiplication mapping from the absolute masses in one possible world to the absolute masses in another possible world. It is true that the Kinematic Shift is standardly formulated in the same way, by adding velocities to the absolute velocities in one world to obtain the velocities in a different world, but, as Maudlin points out in the quote, in principle the Kinematic Shift Argument could have been reformulated to 'absolutely' or 'intrinsically' pick out the world that is at absolute rest, without 'comparing' it to other possible worlds. There is no such reformulation of the Leibniz Scaling.

In the case of absolute masses in fact being empirically meaningful, as I have argued for, we face the problem that we cannot express that knowledge by non-trivially expressing which

\footnotetext{
${ }^{66}$ In fact, absolute masses are underdetermined in a sense that goes beyond even their inexpressibility [22, §2.7]. (Moreover, since comparativist mass ratios are generally also quidditistic-Machian comparativism being the exception [35] - this further underdetermination seems to apply to comparativist mass ratios as well.)
} 


\begin{tabular}{l|l|l} 
& inexpressible & expressible \\
\hline ignorance & $\begin{array}{l}\text { - absolute position } \\
\text { - absolute \& relative speed } \\
\text { (against Maudlin [55]) } \\
\text { - absolute mass, if it would have } \\
\text { been empirically meaningless }\end{array}$ & $\begin{array}{l}\text { - direction of absolute velocity } \\
\text { - being at absolute rest or not }\end{array}$ \\
\hline knowledge & $\begin{array}{l}\text { - absolute mass } \\
\text { - absolute position, if the force law } \\
\text { had depended on position } \\
\text { - absolute rotation }\end{array}$ & $\begin{array}{l}\text { - number of corners } \\
\text { - mass ratios }\end{array}$ \\
\hline
\end{tabular}

Table 1: Examples of expressible and inexpressible knowledge and ignorance.

absolute mass this object has. We can state that it has or does not have the same mass as another object, and we can state that we know that it does not have twice the absolute mass that it actually has, but that is as good as it gets.

This latter case is similar to a Newtonian possible world with a force law that does depend on position. Since this is not true in standard NG, substantivalists often turn to a sophisticated version, which denies primitive identities of spacetime points, but if the force law $(\mathrm{s})$ had in fact depended on position these primitive identities would have been needed for the force(s) to latch onto. Similarly, since absolute masses are empirically meaningful, the magnitudes require non-qualitative identities to which the forces latch on (i.e. these identities are required for the forces to be well-defined/ referring, in the sense of uniquely matching up instances of initial conditions, including masses, with, say, accelerations).

In summary, to require expressible knowledge of absolute masses as criterion for their detectability, or expressible ignorance as criterion for their undetectability, would be incorrect. This would make absolutism in some sense a priori false, which would be strange since detectability it supposed to be something that is to be determined a posteriori. This is basically the naive argument in another guise ( $\S 4)$. Similarly, comparativism is in some sense also $a$ priori false, since there is nothing that one could ever have been ignorant of in the first place. The obvious response, on behalf of the undetectability approach, is that we were never interested in this narrow sense of (un)detectability ("detecting-which"), which requires one to be able to express the specific absolute masses that we are either ignorant of or have knowledge of. The notion of detectability is supposed to line up with the empirical meaningfulness of absolute masses, not to be divorced from it. That changing the absolute masses results in a detectable difference is sufficient for the masses to be detectable ("detecting-that"), even if we cannot detect which specific mass obtains. Similarly, if we know that absolute masses make no detectable difference, then it does not matter that we cannot further specify which absolute mass it is of which we do not know that it obtains. I completely agree. But once we accept this broader notion of detectability, that uniformly changing the masses leads to a detectable difference, that is a detectably different possible world, the undetectability approach has collapsed onto the possibility checking approach - it is not merely equivalent but literally becomes the same approach. Detectability is now an explicitly modal notion, distinct from the non-modal manner in which we detected the number of fingers of a glove. We are now in the business of comparing the detectable features of possible worlds that differ in their distribution of ab- 
solute masses (but not mass ratios). ${ }^{67}$ We are thus naturally pushed towards the possiblity checking approach. This approach focuses merely on checking whether the correct empirically distinct possible worlds are included, not on 'labeling' or 'matching up' those possible worlds with expressible absolute masses.

\section{Solving the Ozma Mass Problem}

It is important to realise the implications of the previous discussion for the evaluation of different variants of the Ozma Mass Problem. The Ozma Mass Problem concerns the question of whether we can convey the meaning of, say, ' $1 \mathrm{~kg}$ ' to the aliens (ideally in the same sense as we can convey dimensionless determinates). So far we have equivocated between three interpretations of the problem. 1) Can we express a quidditistic absolute mass (say the one that we have labelled ' $1 \mathrm{~kg}$ ') to the aliens, in the same direct (i.e. kinematic) sense as we can convey dimensionless determinates? 2) Can the aliens discover (i.e. detect) that absolute masses are empirically relevant? 3) Can we ensure that the aliens pick out a specific object with an absolute mass that is the same as the mass that we have labelled, say, ' $1 \mathrm{~kg}$ '?

The content of kinematic comparativism boils down to a negative answer to the first question. It initially seemed impossible to answer the last two questions affirmatively, for the same reason - the only direct (i.e. non-dynamical) way for the aliens to express the mass of an object is by comparing it to a standard reference mass in their capital. However, the original Ozma Handedness Problem can be solved by asking the aliens to perform a certain experiment (namely any decay that is governed by the weak interactions) to determine unequivocally what (the orientation that we call) left is. We now realise that we have a similar experiment available to provide a positive answer to the last two interpretations of the Ozma Mass Game. ${ }^{68}$ Ask the aliens to set up two particles of equal mass according to the scenario above - the comparativist's bucket - and let them observe whether they escape or not. These experimental instructions suffice to demonstrate to the aliens that absolute masses are empirically relevant (i.e. interpretaion 2) - they can "detect-that" (even if they cannot "detect-which"). The comparativist's bucket experiment allows the mass of these particles to be expressed in a 'natural' mass unit - that is a dynamically privileged mass unit - which is such that 1 unit of mass would just about escape. (Of course, kinematic comparativism tells us that this natural unit cannot be forced upon us. The aliens are still free to use another unit. But what the existence of this dynamically privileged natural unit does allow is an unambiguous way of communicating and comparing units.) In this way, then, we can ensure (given a particular pair of rod and clock) that we can have the aliens pick out a specific object that has the same quidditistic mass as our standard reference object in Paris (interpretation 3) - even though they cannot "detect-which".

This solution is however not as neat as the solution to the Ozma Handedness Problem. For that experiment no other information (other than the type of particle), that is no initial condition, is relevant. In the mass case the answer will depend on the initial distance and initial velocity of the two particles in the experiment. In other words, the mass unit depends on the length and time units. ${ }^{69}$ We have nevertheless made some progress. We have solved the

\footnotetext{
${ }^{67} \S 8.3$ of Dasgupta's 2013 paper [3] suggests that Dasgupta is sympathetic to this way of thinking. $\S 8$ of his 2015 paper [72] suggests otherwise.

${ }^{68}$ Of course we could just describe a specific elementary particle to them and use its mass as the unit of mass, but here we consider NG only.

${ }^{69}$ See also Maxwell's mass unit $[73$, vol.1, §5, 3-4].
} 
Simple Ozma Mass Problem, where we are allowed to send the aliens a rod and clock. In other words, we have reduced the Ozma Mass Problem to the Ozma Length Problem and Ozma Time Problem. The claim that we have defined a natural mass unit is thus to be interpreted as there being a dynamically privileged unit for mass, but only relative to a particular pair of rod and clock.

\section{Conclusion, Outlook \& Scope}

This paper criticised Dasgupta's comparativist formulation of Newtonian Gravity, as encapsulated in L2 (p.16). Dasgupta equates the main premise in the argument for (metaphysical) comparativism about mass, the claim that comparativism is empirically adequate -dynamic comparativism - to showing that absolute masses are undetectable. The first result of this paper was to develop an argument by Baker to the contrary: absolute masses are in fact empirically meaningful, that is detectable (in some weak sense). Dasgupta's analysis of the undetectability, that is the threat to comparativism by Leibniz Mass Scaling scenarios (p.12), contains two mistakes. He misinterprets the ceteris paribus clause in the Leibniz Scaling so as to include forces, whereas the gravitational force of course depends on mass and thereby potentially changes when the masses change uniformly. Additionally, he takes acceleration ratios to be the only detectable data that need to be accounted for, whereas those facts underdetermine what is really observable: (relative) trajectories. Once we correct these mistakes, inter-world Leibniz Mass Scaling scenarios, that is the comparativist's bucket experiment, indicate that absolute masses are empirically meaningful - and thus detectable in at least a very weak sense - since varying them uniformly generates detectably different (relative) trajectories.

The second result was as argument against equating the empirical adequacy of comparativism, (a narrow definition of) the undetectability of absolute masses, and the empirical meaninglessness of absolute masses (i.e. anti-realism about absolute masses) in the first place. The empirical meaninglessness of absolute masses comes apart from the empirical adequacy of comparativism, since comparativism is a claim only about the relative fundamentality of absolute masses, and is thus in principle consistent with their reality. Moreover, if detectability requires one to express which specific quidditistic mass has been detected, and undetectability requires one to express which mass has been failed to detect, then neither absolutism nor comparativism can be proven correct since kinematic comparativism precludes such "detecting-which". If we adopt a broader notion of detectability, that an inter-world Leibniz Mass Scaling - that is an expressible difference in the sets of absolute masses between two possible worlds, even though those masses are not expressible themselves - leads to empirically distinct (relative) trajectories, then detectability has explicitly become a modal notion and collapses into the possibility checking approach. Thus, to the extent that the undetectability approach is distinct from the possibility checking approach it is problematic. To the extent that it is (not merely equivalent but literally) the same, let us simply develop the more perspicuous possibility checking approach in detail, as is done elsewhere [22, Ch.3] [35].

These results are clearly problematic for comparativism, but do not yet rule conclusively in favour of absolutism about mass in Newtonian Gravity. A few possible strategies remain open to the comparativist. A first response is to choose an alternative ${ }^{70}$ to the standard ideology that we have been assuming - distances, velocities and masses. The ceteris paribus clause in

\footnotetext{
${ }^{70}$ That is, yet another alternative besides the ideology proposed by Baker (i.e. distances, velocities and accelerations).
} 
the Leibniz Mass Scaling transformation is ambigious. When keeping the standard ideology the same, a uniform change in masses indeed leads to an empirically distinct evolution. This may not remain true though if one keeps other determinables the same. A simple example: if you change the masses and keep the velocities the same this will change the momenta, but if you instead keep the momenta constant then this will change the velocities. Perhaps it is possible to find exactly the right ideology to ensure that a scaling of masses is compensated in exactly the right way as to produce an empirically identical evolution [22, §4.2.2].

A second strategy to refute the empirical meaningfulness of absolute masses would be to change not the ideology, but the laws of nature. Dasgupta's attempt failed, but another attempt called Machian Comparativism stands a better chance [35].

A third, more compromising approach, saves the letter of comparativism (i.e. mass ratios are not grounded in absolute masses) but gives up its spirit (i.e. anti-realism about objective, nonconventional absolute masses). An instance of this approach would be regularity comparativism, which grounds the absolute masses in mass relations plus other non-conventional non-mass facts, namely a complete four-dimensional mosaic of spatiotemporal relations [6].

A final, completely revisionary approach would be to design a comparativist theory from scratch, which may correspond to a radically distinct set of physically possible worlds than those allowed by standard Newtonian Gravity - as long as it of course does include a model that could (approximately) represent the actual world (in the Newtonian regime) [22, §4.4].

One theme of this paper was that absolutism-comparativism debates are an empirical matter: one determinable in one theory might be absolute, and another determinable in perhaps another theory might be comparative. The results of this paper against comparativism about mass in Newtonian Gravity are thus not an argument against comparativism (about mass) per se. But Newtonian Gravity - and with it most if not all of our current, best theories - is only approximately true of our actual world, which strictly speaking means that it is false. If one then understand this paper's results according to what Williams calls the standard account of theory interpretation, which holds that to give an interpretation of a physical theory is to answer the counterfactual question "if this theory provided a true description of the [actual] world in all respects, what would the world be like?" [74, p.1-2], then this paper has no ramifications for the metaphysics of our actual world. It then merely seems an exercise in developing the methodology relevant to deciding on matters of absolutism vs. comparativism and to chart the potential pitfalls. That is, preparing our metaphysical toolkit for the moment when we have found our final theory. Although I do in fact consider this a worthwhile goal, this paper also goes beyond this goal, and has to do so. As repeatedly mentioned, a crucial bone of contention in this debate is the empirical adequacy of comparativism about mass in NG. This issue and thereby the whole debate is moot without having a well-defined data-set-which both sides agree on - that the theory is trying to account for. And that data-set of course has to pertain to the actual world. I therefore intend the results of this paper to be understood within William's alternative to the standard framework of theory interpretation, according to which interpreting a physical theory is to answer the question "given that this theory provides an approximately true description of our world, what is our world approximately like?" [74, p.2]. The merely approximate truth of the theory of NG is cashed out by focusing only on a proper subset of the empirical data that needs to be accounted for. This Newtonian subset ignores data at small length scales (quantum effects), at speeds comparable to the speed of light (relativistic effects), at small accelerations (MONDian effects), and in strong gravitational fields (generally relativistic effects). That the actual world may only approximately, that is only emergently 
contain absolute Newtonian masses is acknowledged by acknowledging that Newtonian Gravity is not a fundamental theory. To the extent that Newtonian masses then provide an approximate description of our actual world, e.g. at the level of planetary motion, the results of this paper push back against this approximate description being in terms of mass ratios only, that is without absolute masses.

\section{Acknowledgements}

I would like to thank David Baker, Harvey Brown, Adam Caulton, Eddy Keming Chen, Erik Curiel, Shamik Dasgupta, Neil Dewar, Patrick Dürr, Sam Fletcher, Dennis Lehmkuhl, Niels Linnemann, Tushar Menon, Thomas Møller-Nielsen, Zee Perry, Oliver Pooley, Carina Prunkl, John Roberts, Simon Saunders, Syman Stevens, Reinier van Straten, Chris Timpson, Teru Tomas, David Wallace and Alastair Wilson for useful discussions, comments on earlier drafts of this essay, and for their generosity with their time. I am grateful for questions and comments from the audiences at the Ockham Society, the Socrates Society, the DPhil seminar, the Philosophy of Physics Research Seminar and the Philosophy of Physics Graduate Lunch Seminar at the University of Oxford, as well as SOPhiA 2014 in Salzburg, the 2014 Bucharest Graduate Conference in Early Modern Philosophy, the 2014 and 2015 Tübingen Summer Schools in HPS, the 2015 Metaphysics of Quantities Conference at NYU, the 2015 Graduate Workshop in Mathematical Philosophy at the MCMP in Munich, and the DPG2015 in Berlin. This material is based on work supported by the Arts and Humanities Research Council of the UK, a Scatcherd European Scholarship, and in part by the DFG Research Unit "The Epistemology of the Large Hadron Collider" (grant FOR 2063). The major part of this essay was written while I was at Magdalen College and Department of Philosophy, University of Oxford, including a two-month research visit to Princeton University (supported by the AHRC Research Training Support Scheme and a Santander Academic Travel Award).

\section{References}

[1] Eleanor Knox. Newtonian spacetime structure in light of the equivalence principle. British Journal for the Philosophy of Science, 65(4):863-80, 2014.

[2] W.V.O. Quine. Ontology and ideology. Philosophical Studies, 2:183-204, 1951.

[3] Shamik Dasgupta. Absolutism vs comparativism about quantity. In Karen Bennett and Dean W. Zimmerman, editors, Oxford Studies in Metaphysics, volume 8, pages 105-147. Oxford University Press, 2013.

[4] John T. Roberts. A case for comparativism about physical quantities. Manuscript, accessed on 20 Sept 2016, https://www.academia.edu/28548115/A_Case_for_Comparativism_ about_Physical_Quantities_--_SMS_2016_Geneva.

[5] Theodore Sider. The tools of metaphysics and the metaphysics of science. Draft of 16 August 2018.

[6] Niels C.M. Martens. Regularity comparativism about mass in newtonian gravity. Philosophy of Science, 84(5):1226-1238, 2017. 
[7] W.A. Bauer. An argument for the extrinsic grounding of mass. Erkenntnis, 74(1):81-99, 2011.

[8] Ned Hall. Humean reductionism about laws of nature. Manuscript, http://philpapers . org/archive/HALHRA.pdf.

[9] Barry Loewer. Humean supervenience. Philosophical Topics, 24(1):101-127, 1996.

[10] Michael Esfeld. Quantum humeanism, or: physicalism without properties. The Philosophical Quarterly, 64(256):453-470, 2014.

[11] Michael Esfeld, Dustin Lazarovici, Vincent Lam, and Mario Hubert. The physics and metaphysics of primitive stuff. British Journal for the Philosophy of Science, 68(1):133$161,2017$.

[12] Michael Esfeld, Dirk-André Deckert, and Andrea Oldofredi. What is matter? the fundamental ontology of atomism and structural realism. forthcoming in Anna Ijjas and Barry Loewer (eds.) A guide to the philosophy of cosmology, Oxford University Press.

[13] Michael Esfeld and Dirk-André Deckert. A Minimalist Ontology of the Natural World. New York: Routledge, 1st edition, 2017.

[14] Antonio Vassallo, Dirk-André Deckert, and Michael Esfeld. Relationalism about mechanics based on a minimalist ontology of matter. European Journal for Philosophy of Science, pages $1-20,2016$.

[15] Mario Hubert. Quantity of matter or intrinsic property: Why mass cannot be both. In Laura Felline, Antonio Ledda, Francesco Paoli, and Emanuele Rossanese, editors, New Developments in Logic and Philosophy of Science, volume 3, pages 267-77. London: College Publications, 2016.

[16] D. Armstrong. A Theory of Universals: Volume 2. Cambridge: Cambridge University Press, 1978.

[17] D. Armstrong. Are quantities relations? a reply to bigelow and pargetter. Philosophical Studies, 54:305-316, 1988.

[18] Brent Mundy. The metaphysics of quantity. Philosophical Studies, 51(1):29-54, 1987.

[19] D. Lewis. Philosophical Papers, volume ii. Oxford University Press, 1986.

[20] E. Mach. The Science of Mechanics. The Open Court Publishing Co., translated by T.J. McCormack, 1960 [1893].

[21] David J. Baker. Some consequences of physics for the comparative metaphysics of quantity. Manuscript, May 2013.

[22] Niels C.M. Martens. Against Comparativism about Mass in Newtonian Gravity - a Case Study in the Metaphysics of Scale. PhD thesis, Magdalen College, University of Oxford, 2017.

[23] Hartry H. Field. Science without Numbers: A Defence of Nominalism. Oxford: Basil Blackwell, 1980. 
[24] H. Field. Can we dispense with space-time? In PSA 2, 1985.

[25] Bertrand Russell. The Principles of Mathematics. W.W. Norton \& Company, 1903.

[26] B. Ellis. Basic Concepts of Measurement. Cambridge: Cambridge University Press, 1966.

[27] J. Bigelow, R. Pargetter, and D.M. Armstrong. Quantities. Philosophical Studies, 54:287316, 1988.

[28] J. Bigelow and R. Pargetter. Science and Necessity. Cambridge: Cambridge University Press, 1990.

[29] Frank Arntzenius. Space, Time, \& Stuff. Oxford University Press, 2012.

[30] Maya Eddon. Quantitative properties. Philosophy Compass, 8(7):633-645, 2013.

[31] Zee Perry. Physical Quantities: Mereology and Dynamics. PhD thesis, 2016.

[32] Marco Dees. Physical magnitudes. Pacific Philosophical Quarterly, 99:817-841, 2018.

[33] N.C.M. Martens. The metaphysics of emergent spacetime theories. Manuscript.

[34] Niels C.M. Martens. Against laplacian reduction of newtonian mass to spatiotemporal quantities. Foundations of Physics, 48(5):591-609, 2018.

[35] Niels C.M. Martens. Machian comparativism about mass. Forthcoming in The British Journal for the Philosophy of Science.

[36] M. Gardner. The New Ambidextrous Universe: Symmetry and Asymmetry from Mirror Reflections to Superstrings. Mineola, New York: Dover Publications, Inc, dover 2005 (= third) edition, 1964 [revised and retitled in 1990].

[37] F. Dowker. Causal sets and the deep structure of spacetime. arXiv:gr-qc/0508109v1, 2008.

[38] H. Weyl. Philosophy of Mathematics and Natural Science. Princeton: Princeton University Press, 1949.

[39] Max Jammer. Concepts of Mass in Contemporary Physics and Philosophy. Princeton University Press, 2000.

[40] Nick Huggett. Space from Zeno to Einstein: Classic Readings with a Contemporary Commentary. Cambridge, MA: MIT Press, 1999.

[41] James van Cleve. Right, left, and the fourth dimension. The Philosophical Review, 96:3368, 1987. Reprinted in [75, p.203-234], 1991.

[42] Franz Brentano. Psychology from an Empirical Standpoint. 1973.

[43] Jonathan Bennett. The difference between right and left. American Philosophical Quarterly, 7:175-191, 1970. Reprinted in [75, p.97-130], 1991.

[44] John Earman. Kant, incongruous counterparts, and the nature of space and space-time. In In [75, p.131-149]. 1991. 
[45] J. Van Cleve. Introduction to the arguments of 1770 and 1783. In [75, p.15-26]. 1991.

[46] Shamik Dasgupta. On the plurality of grounds. Philosophers' Imprint, 14(20):1-28, 2014.

[47] H. Zanstra. A study of relative motion in connection with classical mechanics. Physical Review, 23(4):528-545, 1924.

[48] Isaac Newton. Philosophiae Naturalis Principia Mathematica. London: Joseph Streater, 1686/7. The Scholium to the Definitions has been reprinted in Alexander [54].

[49] J. Barbour. The end of time: The next revolution in physics. Oxford: Oxford University Press, 2000.

[50] Simon Saunders. Mirroring as an a priori symmetry. Philosophy of Science, 74:452-480, 2007.

[51] Gordon Belot. Rehabilitating relationalism. International Studies in the Philosophy of Science, 13(1):35-52, 1999.

[52] Gordon Belot. Geometry and motion. British Journal for the Philosophy of Science, 51(4):561-595, 2000.

[53] Gordon Belot and John Earman. Pre-socratic quantum gravity. In Craig Callendar and Nick Huggett, editors, Physics meets philosophy at the Planck scale: Contemporary theories in quantum gravity, chapter 10, pages 213-55. Cambridge University Press, 2001.

[54] H.G. Alexander, editor. The Leibniz-Clarke Correspondence. Manchester: Manchester University Press, 1956 [1717]. Originally written by Leibniz, G.W. \& Clarke, S. in 1715-16 and published by Clarke, S. in 1717.

[55] Tim Maudlin. Buckets of water and waves of space: Why spacetime is probably a substance. Philosophy of Science, 60:183-203, 1993.

[56] Shamik Dasgupta. Symmetry as an epistemic notion (twice over). The British Journal for the Philosophy of Science, 67:837-878, 2016.

[57] Thomas Møller-Nielsen and James Read. Redundant epistemic symmetries.

[58] David J. Baker. Comparativism with mixed relations. June 2013 Manuscript.

[59] R. DiSalle. Space and time: Inertial frames. Stanford Encylopedia of Philosophy, 2002 [revised in 2009].

[60] Moritz Schlick. Space and time in contemporary physics: An introduction to the theory of relativity and gravitation. see mulder and van de velde-schlick [76, ch.9, p. 207-269]. 1979 [1922]. Translated by Henry Brose and Peter Heath from Raum und zeit in der gegenwärtigen physik, 4th edition, Berlin.

[61] Moritz Schlick. General Theory of Knowledge. Library of Exact Philosophy Series. Springer-Verlag, 1974 [1925]. Translated by Albert Blumberg, from the 2nd German Edition of Allgemeine Erkenntnislehre, Berlin. 
[62] Moritz Schlick. Philosophy of Nature. Philosophy Library, Inc., 1949. Translated by Amethe von Zeppelin.

[63] Syman Stevens. The Dynamical Approach to Relativity as a Form of Regularity Relationalism. PhD thesis, University of Oxford, 2014.

[64] Bradford Skow. Sklar's maneuver. British Journal for the Philosophy of Science, 58:777$786,2007$.

[65] Oliver Pooley. The Reality of Spacetime. Manuscript.

[66] F. Arntzenius. Are there really instantaneous velocities? The Monist, 83(2):187-208, 2000.

[67] E. Grant. Physical Science in the Middle Ages. Wiley, New York and London, 1981.

[68] E.J. Dijksterhuis. The Mechanisation of the World Picture. Princeton: Princeton University Press, 1986.

[69] Daniel C. Dennett. Real patterns. The Journal of Philosophy, 88(1):27-51, 1991.

[70] Shamik Dasgupta. Inexpressible ignorance. Philosophical Review, 124(4), 2015.

[71] David Lewis. Ramseyan humility. In David Braddon-Mitchell and Robert Nola, editors, Conceptual Analysis and Philosophical Naturalism, pages 203-22. Cambridge, MA: MIT Press, 2009.

[72] Shamik Dasgupta. Substantivalism vs relationalism about space in classical physics. Philosophy Compass, 10(9):601-624, 2015.

[73] James Clerk Maxwell. A treatise on electricity and magnetism, Vol. 1 \& 2. Cambridge: Cambridge University Press, 1873.

[74] Porter Williams. Scientific realism made effective. Forthcoming in The British Journal for the Philosophy of Science, pages 1-29, 2018.

[75] J. Van Cleve and R.E. Frederick, editors. The Philosophy of Left and Right: Incongruent Counterparts and the Nature of Space. Dordrecht, the Netherlands: Kluwer Academic Publishers, 1991.

[76] Henk Mulder and Barbara van de Velde-Schlick, editors. Moritz-Schlick: Philosophical Papers, volume 11-I (1909-1922) of Vienna Circle Collection Series. Reidel Publishing Company, 1979. 